\title{
Superstable Interactions in Classical Statistical Mechanics
}

\author{
D. RUELLE
}

I.H.E.S. Bures-sur-Yvette, France

Received March 11, 1970

\begin{abstract}
We consider classical systems of particles in $v$ dimensions. For a very large class of pair potentials (superstable lower regular potentials) it is shown that the correlation functions have bounds of the form

$$
\varrho\left(x_{1}, \ldots, x_{n}\right) \leqq \xi^{n} .
$$

Using these and further inequalities one can extend various results obtained by Dobrushin and Minlos [3] for the case of potentials which are non-integrably divergent at the origin. In particular it is shown that the pressure is a continuous function of the density. Infinite system equilibrium states are also defined and studied by analogy with the work of Dobrushin [2a] and of Lanford and Ruelle [11] for lattice gases.
\end{abstract}

\section{Introduction}

A number of papers have been devoted to the study of the thermodynamic limit (infinite volume limit) in the statistical mechanics of classical systems of particles in $v$ dimensions. Fairly satisfactory results have been obtained for the thermodynamic functions: existence of the limit, convexity (stability) properties, and the equivalence of the various ensembles ${ }^{1}$. For other problems (continuity of the pressure as a function of specific volume, study of correlation functions) the results are less satisfactory due to a technical difficulty: it is hard to exclude large fluctuations of the number of particles in a small region of space. It is true that if many particles are put in a small region $\Delta$ of space their repulsion will lead to a large positive potential energy (and therefore to a small probability in the grand canonical ensemble), but it is difficult to estimate the interaction energy of the particles in $\Delta$ with the neighbouring ones. In the present paper we solve the technical difficulty just mentioned and study some consequences of the solution.

${ }^{1}$ See the pioneering work of Van Hove [15], Yang and Lee [16] and the articles of Ruelle [13], Fisher [5], Griffiths [7]. For a general exposition and further references see Ruelle [14]. 
We shall assume that the interaction between particles is given by a pair potential $\Phi^{2}$, i.e. the energy of $m$ particles located at $x_{1}, \ldots, x_{m}$ is

$$
U\left(x_{1}, \ldots, x_{m}\right)=\sum_{i<j} \Phi\left(x_{j}-x_{i}\right)
$$

where $\Phi$ is a Lebesgue measurable function which satisfies $\Phi(x)=\Phi(-x)$ and which may take real values and the value $+\infty$. In order to have a system with thermodynamic behaviour, we assume that $\Phi$ is stable, i.e. there exists $B \geqq 0$ such that, for all $m, x_{1}, \ldots, x_{m}$,

$$
U\left(x_{1}, \ldots, x_{m}\right) \geqq-m B .
$$

If $\Phi$ is of the form $\Phi=\Phi^{\prime}+\Phi^{\prime \prime}$ where $\Phi^{\prime}$ is a stable pair potential and $\Phi^{\prime \prime}$ is a positive continuous function with $\Phi^{\prime \prime}(0)>0$, we say that $\Phi$ is superstable ${ }^{3}$. The reader may convince himself that stable potentials which are not superstable are in a sense exceptional: in fact the only case of interest is $\Phi=0$. If $\Lambda$ is a fixed bounded region of $\mathbb{R}^{v}$, and $\Phi$ a superstable potential there exist $A>0$ and $B \geqq 0$ such that for all $m$ and all $x_{1}, \ldots, x_{m} \in \Lambda$,

$$
U\left(x_{1}, \ldots, x_{m}\right) \geqq A m^{2}-B m \text {. }
$$

We say that $\Phi$ is lower regular if there is a positive decreasing function $\varphi$ on $[0,+\infty)$ such that

and for all $x \in \mathbb{R}^{v}$

$$
\int_{0}^{\infty} t^{\nu-1} d t \varphi(t)<+\infty
$$

$$
\Phi(x) \geqq-\varphi(|x|) .
$$

We summarize now in two theorems the main results of Sections 1-4 of the present paper

0.1 . Theorem ${ }^{4}$. Let $\Phi$ be a superstable and lower regular pair potential, let $\Lambda$ be a bounded Lebesgue measurable region in $\mathbb{R}^{v}$, and let

$$
Z=\sum_{n=0}^{\infty} \frac{z^{n}}{n !} \int d x_{1} \ldots d x_{n} e^{-\beta U\left(x_{1}, \ldots, x_{n}\right)}
$$

be the grand partition function at activity $z$.

${ }^{2}$ Actually, a weaker assumption is made in Sections 1 and 2.

3 A slightly less restrictive definition is given in Section 1.

${ }^{4}$ See Proposition 2.6 and Corollary 2.9. Part (a) of the theorem is an easy result for positive or hard core pair potentials (see [14], Exercise 4.D). Part (b) had been proved by Dobrushin and Minlos [3] for pair potentials which are non-integrably divergent at the origin. 
(a) Define the correlation functions by

$\varrho_{\Lambda}\left(x_{1}, \ldots, x_{m}\right)=Z^{-1} \sum_{n=m}^{\infty} \frac{z^{n}}{(n-m) !} \int_{\Lambda^{n-m}} d x_{m+1} \ldots d x_{n} e^{-\beta U\left(x_{1}, \ldots, x_{n}\right)}$.

There exists a positive constant $\xi$, independent of $\Lambda, m, x_{1}, \ldots, x_{m}$, such that

$$
\varrho_{\Lambda}\left(x_{1}, \ldots, x_{m}\right) \leqq \xi^{m} .
$$

(b) Given $\lambda>0$, there exist $g>0$ and $d \geqq 0$ such that if $\Delta$ is any bounded Lebesgue measurable subset of $\mathbb{R}^{v}$ with diameter $L \geqq \lambda$, then the grand canonical probability of finding more than $m$ particles in $\Delta$ is less than

$$
\exp \left[-g \frac{m^{2}}{L^{v}}+d m\right]
$$

The probability in question is

$Z^{-1} \sum_{n=m}^{\infty} \sum_{p=0}^{\infty} \frac{z^{n+p}}{n ! p !} \int_{(\Lambda \cap \Delta)^{n}} d x_{1} \ldots d x_{n} \int_{(\Lambda \backslash \Delta)^{p}} d x_{n+1} \ldots d x_{n+p} e^{-\beta U\left(x_{1}, \ldots, x_{n+p}\right)}$.

0.2 . Theorem ${ }^{5}$. Let $\Phi$ be a superstable and lower regular pair potential.

(a) Let $\Lambda$ be a parallelepiped with sides $a^{1}, \ldots, a^{v}$ and volume $|\Lambda|=a^{1} \ldots a^{v}$. If $a^{1}, \ldots, a^{v} \rightarrow \infty$, then $|\Lambda|^{-1} \log Z$ tends to a finite limit $\beta p$ ( $p$ is the thermodynamic limit of the grand canonical pressure).

(b) Assume that

$$
\int d x\left|1-e^{-\beta \Phi(x)}\right|<+\infty
$$

then, the pressure $p$ defined in (a) is a continuous function of the density $\varrho=\beta z \frac{\partial p}{\partial z}$ (inside of its interval of definition).

In Section 5 we study the infinite volume limit of correlation functions. One does not expect that this limit will be unique (because of the possible occurrence of phase transitions), but the following result is obtained.

0.3. Theorem ${ }^{6}$. Let $\Phi$ be a superstable and lower regular pair potential; assume that

$$
\int d x\left|1-e^{-\beta \Phi(x)}\right|<+\infty .
$$

${ }^{5}$ See Theorem 3.3 and Theorem 5.3. Part (b) of the present theorem was known in the extreme cases of pair potentials bounded from above (Ruelle [13]) or very repulsive at the origin (positive or hard core: Penrose and Ginibre [6]; non integrably divergent: Dobrushin and Minlos [3]). An attempt at bridging the gap between the two cases started the work presented in this article.

${ }^{6}$ See Theorem 5.5 and Corollary 5.3.

9 Commun. math. Phys., Vol. 18 
Let $\left(\Lambda_{j}\right)$ be a sequence of bounded Lebesgue measurable regions of $\mathbb{R}^{v}$, tending to infinity in the sense that for every bounded $\Delta$ there exists $n_{\Delta}$ such that $\Delta \subset \Lambda_{j}$ if $j \geqq n_{\Delta}$. Then one can choose a subsequence $\left(\Lambda_{k}^{\prime}\right)$ of $\left(\Lambda_{j}\right)$ such that

$$
\lim _{k \rightarrow \infty} \varrho_{\Lambda_{k}}\left(x_{1}, \ldots, x_{m}\right)=\varrho\left(x_{1}, \ldots, x_{m}\right)
$$

uniformly on the bounded subsets of $\left(\mathbb{R}^{v}\right)^{m}$. The infinite volume correlation functions obtained as limit satisfy the Kirkwood-Salsburg equations

$$
\begin{aligned}
& \varrho\left(x_{1}, \ldots, x_{m}\right)=z e^{-\beta \sum_{i=2}^{m} \Phi\left(x_{i}-x_{1}\right)} \sum_{n=0}^{\infty} \frac{1}{n !} \int d x_{m+1} \ldots d x_{m+n} \\
& {\left[\prod_{j=1}^{n}\left(e^{-\beta \Phi\left(x_{m}+j-x_{1}\right)}-1\right)\right] \varrho\left(x_{2}, \ldots, x_{m+n}\right) .}
\end{aligned}
$$

The other results of Section 5 are of a less elementary nature and are only outlined here. A concept of infinite volume equilibrium state is introduced. Such a state may be described by correlation functions satisfying the Kirkwood-Salsburg equations. It may also be described by a probability measure $\mu$ (on a suitable measurable space $\mathscr{X}$ ) satisfying "equilibrium equations" of a type already known for lattice systems (Dobrushin [2a], Landford and Ruelle [11]). The set $\Sigma$ of (infinite volume) equilibrium states is non empty, convex and compact $^{7}$ and a Choquet simplex ${ }^{8}$. The last statement means that every equilibrium state may, in a unique manner, be decomposed into extremal equilibrium states. If this decomposition is non trivial for a pure thermodynamic phase we have an example of symmetry breakdown ${ }^{9}$. Let $\Sigma_{0}$ be the set of equilibrium states which are invariant under the effect of translations of $\mathbb{R}^{v}$. This set is non empty, convex, and compact. It is again a Choquet simplex and this fact has the physical interpretation that every invariant equilibrium state has a unique decomposition into pure thermodynamic phases.

For sufficiently small activity, $\Sigma$ consists of just one point. In that case the thermodynamic limit of the correlation functions is unique ${ }^{10}$. In general from every sequence $\left(\Lambda_{n}\right)$ of bounded regions of $\mathbb{R}^{v}$ tending to infinity one can extract a subsequence such that the corresponding correlation functions tend to some point of $\Sigma^{11}$.

\footnotetext{
7 With respect to the topology of uniform convergence on compacts of the correlation functions.

${ }^{8}$ See for instance Choquet and Meyer [2].

9 For a discussion of these concepts, see [14] Chapters 6 and 7, Lanford and Ruelle [11].

10 This was known, see for instance [14] Section 4.2. References are given in [14] to the original papers of Ruelle and Penrose. The author has recently become aware of earlier work (for positive potentials) by Bogoljubov and Khatset [1].

11 This may not give all points of $\Sigma$. One can however get all points of $\Sigma$ by introducing suitable "boundary effects", namely by prescribing suitable distributions of particles outside of the $\Lambda_{n}$.
} 


\section{Conditions on the Interaction}

For all integers $m \geqq 0$ and all $x_{1}, \ldots, x_{m} \in \mathbb{R}^{v}$, let $U\left(x_{1}, \ldots, x_{m}\right) \in \mathbb{R}$ $\cup\{+\infty\} ; U$ is thus a function on $\sum_{m}\left(\mathbb{R}^{v}\right)^{m}$. We say that $U$ is an interaction if it satisfies the following conditions.

$\left(\mathrm{I}_{1}\right)$ Measurability. For each $m,\left(x_{1}, \ldots, x_{m}\right) \rightarrow U\left(x_{1}, \ldots, x_{m}\right)$ is Lebesgue measurable.

$\left(\mathrm{I}_{2}\right)$ Permutation invariance.

$$
U\left(x_{i_{1}}, \ldots, x_{i_{m}}\right)=U\left(x_{1}, \ldots, x_{m}\right)
$$

for every permutation $(1, \ldots, m) \rightarrow\left(i_{1}, \ldots, i_{m}\right)$.

$\left(\mathrm{I}_{3}\right)$ Translation invariance.

$$
U\left(x_{1}+a, \ldots, x_{m}+a\right)=U\left(x_{1}, \ldots, x_{m}\right)
$$

for all $a \in \mathbb{R}^{v}$.

( $\left.\mathrm{I}_{4}\right)$ If $y_{1}, \ldots, y_{n} \in \mathbb{R}^{v}$ and $U\left(x_{1}, \ldots, x_{m}\right)=+\infty$, then

$$
U\left(x_{1}, \ldots, x_{m}, y_{1}, \ldots, y_{n}\right)=+\infty \text {. }
$$

$\left(I_{5}\right)$ Normalization. If $m=0$ or $m=1$, then $U\left(x_{1}, \ldots, x_{m}\right)=0$.

We say that the interaction $U$ is stable if it satisfies the condition

(S) There exists $B \geqq 0$ such that for all $m, x_{1}, \ldots, x_{m}$,

$$
U\left(x_{1}, \ldots, x_{m}\right) \geqq-m B .
$$

Let $0<\lambda \in \mathbb{R}$. For every $r \in \mathbb{Z}^{v}$ we define a cube

$$
\mathscr{Q}(r)=\left\{x \in \mathbb{R}^{v}:\left(r^{i}-\frac{1}{2}\right) \lambda \leqq x^{i}<\left(r^{i}+\frac{1}{2}\right) \lambda\right\} .
$$

These cubes form a partition of $\mathbb{R}^{v}$. If $X \in\left(\mathbb{R}^{v}\right)^{m}$, we let $n(X, r)$ be the number of points of the sequence $X=\left(x_{1}, \ldots, x_{m}\right)$ which belong to $\mathscr{Q}(r)$.

A condition stronger than stability is the following

(SS) Superstability. There exist $A>0, B \geqq 0$ such that if $\mathscr{R}$ is a finite subset of $\mathbb{Z}^{v}$ and

$$
x_{1}, \ldots, x_{m} \in \cup_{r \in \mathscr{R}} \mathscr{Q}(r), \quad X=\left(x_{1}, \ldots, x_{m}\right)
$$

then

$$
U(X) \geqq \sum_{r \in \mathscr{R}}\left[A n(X, r)^{2}-B n(X, r)\right] .
$$

Notice that a positive interaction is stable, that the sum of two stable interactions is stable, and that the sum of a stable and a superstable interactions is superstable. 
We write

$$
\begin{gathered}
X=\left(x_{1}, \ldots, x_{m}\right), \quad Y=\left(y_{1}, \ldots, y_{n}\right), \\
X Y=\left(x_{1}, \ldots, x_{m}, y_{1}, \ldots, y_{n}\right) .
\end{gathered}
$$

Let $W(X, Y)$ satisfy

$$
\begin{gathered}
U(X Y)=U(X)+U(Y)+W(X, Y), \\
W(X, Y)=+\infty \quad \text { if } \quad U(X Y)=+\infty
\end{gathered}
$$

In view of $\left(\mathrm{I}_{2}\right),\left(\mathrm{I}_{4}\right)$, these conditions determine entirely the function $W$ : $\left(\sum_{m}\left(\mathbb{R}^{v}\right)^{m}\right) \times\left(\sum_{m}\left(\mathbb{R}^{v}\right)^{m}\right) \rightarrow \mathbb{R} \cup\{+\infty\}$.

If $r \in \mathbb{Z}^{v}$, we let $|r|=\sup _{i}\left|r^{i}\right|$. We say that the interaction $U$ is lower regular if it satisfies the following condition

(LR) There exists a decreasing positive function $\Psi$ on the positive integers such that

$$
\sum_{r \in \mathbb{Z}^{v}} \Psi(|r|)<+\infty
$$

Furthermore if $\mathscr{R}, \mathscr{S}$ are finite subsets of $\mathbb{Z}^{v}$ and

then

$$
\begin{array}{ll}
x_{1}, \ldots, x_{m} \in \cup_{r \in \mathscr{R}} \mathscr{Q}(r), & X=\left(x_{1}, \ldots, x_{m}\right) \\
y_{1}, \ldots, y_{n} \in \cup_{s \in \mathscr{S}} \mathscr{Q}(s), & Y=\left(y_{1}, \ldots, y_{n}\right)
\end{array}
$$

$$
W(X, Y) \geqq-\sum_{r \in \mathscr{R}} \sum_{s \in \mathscr{S}} \Psi(|s-r|)\left[\frac{1}{2} n(X, r)^{2}+\frac{1}{2} n(Y, s)^{2}\right] .
$$

We mention without proof the following easily verified result.

1.1. Proposition. The conditions (SS) and (LR) are invariant under linear transformations of $\mathbb{R}^{v}$ (in particular they are translation invariant and independent of the choice of $\lambda$ ).

We indicate now criteria under which (S), (SS), (LR) hold for interactions associated with pair potentials. A pair potential is a Lebesgue measurable function $\Phi: \mathbb{R}^{v} \rightarrow \mathbb{R} \cup\{+\infty\}$ such that $\Phi(-x)=\Phi(x)$; an interaction $U_{\Phi}$ is defined by

$$
U_{\Phi}\left(x_{1}, \ldots, x_{n}\right)=\sum_{i<j} \Phi\left(x_{j}-x_{i}\right)
$$

Clearly $U_{\Phi} \geqq 0$ if $\Phi \geqq 0$. We say that $\Phi$ is stable (resp. superstable, lower regular) if $U_{\Phi}$ satisfies (S) (resp. (SS), (LR)). Propositions 1.2, 1.3, 1.4 below give criteria for stability, superstability and lower regularity. For proofs, see [14], Section 3.2. 
1.2. Proposition. (a) If $\Phi^{\prime}$ is the Fourier transform of a positive measure with finite total mass, then $\Phi^{\prime}$ is stable.

(b) If $\Phi^{\prime \prime}$ is continuous $\geqq 0$ and $\Phi^{\prime \prime}(0)>0$, then $\Phi^{\prime \prime}$ is superstable.

In particular if $\Phi \geqq \Phi^{\prime}$, then $\Phi$ is stable ${ }^{12}$. If $\Phi$ is stable, then $\Phi+\Phi^{\prime \prime}$ is superstable.

1.3. Proposition. Let $\varphi:[0,+\infty) \rightarrow \mathbb{R}$ be positive, decreasing, and let

$$
\int_{0}^{\infty} t^{\nu-1} d t \varphi(t)<+\infty
$$

If $\Phi(\ddot{x}) \geqq-\varphi(|x|)$ for all $x$, then $\Phi$ is lower regular.

1.4. Proposition (Dobrushin, Fisher, Ruelle). Let $0<d_{1}<d_{2}<+\infty$ and let

$$
\varphi_{1}:[0, d] \rightarrow \mathbb{R} \cup\{+\infty\}, \quad \varphi_{2}:\left[d_{2},+\infty\right) \rightarrow \mathbb{R}
$$

be positive, decreasing and such that

$$
\int_{0}^{d_{1}} t^{\nu-1} d t \varphi_{1}(t)=+\infty, \quad \int_{d_{2}}^{\infty} t^{\nu-1} d t \varphi_{2}(t)<+\infty .
$$

If the pair potential $\Phi$ is bounded below and satisfies

$$
\begin{array}{rll}
\Phi(x) \geqq \varphi_{1}(|x|) & \text { for } & |x| \leqq d_{1}, \\
|\Phi(x)| \leqq \varphi_{2}(|x|) & \text { for } & |x| \geqq d_{2}
\end{array}
$$

then $\Phi$ is superstable and lower regular.

\section{Probability Estimates}

In this section we shall obtain bounds on the correlation functions and other quantities of interest (probability estimates). We assume that the distribution of $X$ is given by the grand canonical ensemble and that the interaction $U$ is superstable and lower regular.

Let $\Lambda$ be a bounded Lebesgue measurable subset of $\mathbb{R}^{v}$ with measure $|\Lambda|>0$, let $U$ be a stable interaction and let $\beta>0, z>0$. The grand canonical probability measure is defined on $\sum_{n \geqq 0} \Lambda^{n}$ by its restriction

to every $\Lambda^{n}$; here

$$
Z^{-1} \frac{z^{n}}{n !} e^{-\beta U\left(x_{1}, \ldots, x_{n}\right)} d x_{1} \ldots d x_{n}
$$

$$
Z=\sum_{n \geqq 0} \frac{z^{n}}{n !} \int_{A^{n}} d x_{1} \ldots d x_{n} e^{-\beta U\left(x_{1}, \ldots, x_{n}\right)} .
$$

12 There exist stable potentials which are not of this type (S. Sherman, private communication). 
The convergence of $Z$ is ensured by the stability of $U$. The probability estimates (Propositions 2.6 and 2.7) will be preceded by technical results, Propositions 2.1 and 2.5 .

Given $\alpha>0$, we can choose an integer $P_{0}>0$ and for each $j \geqq P_{0}$ an integer $l_{j}>0$ such that

$$
\left|\frac{l_{j+1}}{l_{j}}-(1+2 \alpha)\right|<\alpha .
$$

We use the notation ${ }^{13}$

$$
\begin{gathered}
|r|=\sup \left|r^{i}\right|, \\
{[j]=\left\{r \in \mathbb{Z}^{v}:|r| \leqq l_{j}\right\}, \quad[k \backslash j]=[k] \backslash[j],} \\
V_{j}=\left(2 l_{j}+1\right)^{v} .
\end{gathered}
$$

2.1. Proposition. Let $A>0, B \geqq 0$, and let $\Psi$ be a decreasing positive function on the positive integers such that

$$
\sum_{r \in \mathbb{Z}^{v}} \Psi(|r|)<+\infty
$$

If $\alpha$ is sufficiently small one can choose an increasing sequence $\left(\psi_{j}\right)$ such that $\psi_{j} \geqq 1, \psi_{j} \rightarrow \infty$, and fix $P>P_{0}$ so that the following is true.

Let $n(\cdot)$ be a function from $\mathbb{Z}^{v}$ to the integers $\geqq 0$. Suppose that there exists $q$ such that $q \geqq P$ and $q$ is the largest integer for which

Then

$$
\sum_{r \in[q]} n(r)^{2} \geqq \psi_{q} V_{q}
$$

$$
\begin{gathered}
-\sum_{r \in[q+1]}\left[A n(r)^{2}-B n(r)\right]+\sum_{r \in[q+1]} \sum_{s \notin[q+1]} \Psi(|s-r|)\left[\frac{1}{2} n(r)^{2}+\frac{1}{2} n(s)^{2}\right] \\
\leqq-\frac{A}{2} \sum_{r \in[q+1]} n(r)^{2} .
\end{gathered}
$$

$$
\left[\sum_{r} \Psi(|r|)\right] \times\left[(1+3 \alpha)^{2 v+2}-1\right] \leqq \frac{A}{4} .
$$

There exists an increasing function $\psi$ on the positive integers such that

$$
\begin{gathered}
\psi \geqq 1, \quad \lim _{l \rightarrow \infty} \psi(l)=+\infty, \\
\frac{\psi(l+1)}{\psi(l)} \leqq \frac{l+1}{l}
\end{gathered}
$$

${ }^{13}$ The inverted slant $(\backslash)$ denotes set difference. 
and

$$
\sum_{r \in \mathbb{Z}^{\nu}} \psi(|r|) \Psi(|r|)<+\infty .
$$

[Choose $\psi^{*}$ satisfying (2.11) and (2.13), then let $\psi$ be the largest function $\leqq \psi^{*}$ satisfying (2.12).] We define $\psi_{j}=\psi\left(l_{j}\right)$, thus

$$
1 \leqq \frac{\psi_{j+1}}{\psi_{j}} \leqq \frac{l_{j+1}}{l_{j}} \leqq 1+3 \alpha .
$$

The choice of $P$ is made so that Lemma 2.4 below holds. Before proving the proposition we introduce the definition

$$
\Psi_{k}=\sup _{r \in[q+1], s \notin[q+k+1]} \Psi(|s-r|)=\Psi\left(l_{q+k+1}-l_{q+1}+1\right)
$$

and we state a few lemmas.

2.2. Lemma. Let the conditions of Proposition 2.1 be satisfied by $n(\cdot)$ and $q$, and let $k \geqq 0$, then

(a) $\sum_{s \in[q+k \mid q]} n(s)^{2} \leqq\left(\psi_{q+k} V_{q+k}-\psi_{q} V_{q}\right)$,

(b) if $\Psi_{k}$ is defined by (2.15)

$$
\sum_{k=1}^{\infty} \Psi_{k} \sum_{s \in[q+k+2 \backslash q+k+1]} n(s)^{2} \leqq \sum_{k=1}^{\infty}\left(\Psi_{k}-\Psi_{k+1}\right) \psi_{q+k+2} V_{q+k+2} .
$$

(a) follows from the fact that $q$ - is the largest integer for which (2.8) holds; (b) is obtained, using (a), as follows

$$
\begin{aligned}
& \sum_{k=1}^{\infty} \Psi_{k} \sum_{s \in[q+k+2 \backslash q+k+1]} n(s)^{2} \\
= & \sum_{k=1}^{\infty}\left(\Psi_{k}-\Psi_{k+1}\right) \sum_{s \in[q+k+2 \backslash q+2]} n(s)^{2} \\
\leqq & \sum_{k=1}^{\infty}\left(\Psi_{k}-\Psi_{k+1}\right)\left(\Psi_{q+k+2} V_{q+k+2}-\psi_{q} V_{q}\right) \\
\leqq & \sum_{k=1}^{\infty}\left(\Psi_{k}-\Psi_{k+1}\right) \psi_{q+k+2} V_{q+k+2} .
\end{aligned}
$$

\subsection{Lemma.}$$
\text { (a) } \frac{\psi_{j+1} V_{j+1}}{\psi_{j} V_{j}} \leqq(1+3 \alpha)^{v+1} \text {, }
$$$$
\text { (b) } \frac{\psi_{j+2} V_{j+2}-\psi_{j} V_{j}}{\psi_{j} V_{j}} \leqq(1+3 \alpha)^{2 v+2}-1 \text {. }
$$ 
(c) Let $k \geqq 1$, then

$$
\frac{\psi_{q+k+2} V_{q+k+2}}{\psi\left(l_{q+k+1}-l_{q+1}+1\right)\left(2 l_{q+k+1}-2 l_{q+1}+3\right)^{v}} \leqq\left[\alpha^{-1}(1+\alpha)(1+3 \alpha)\right]^{v+1}
$$

Proof of (a).

$$
\frac{\psi_{j+1} V_{j+1}}{\psi_{j} V_{j}} \leqq \frac{l_{j+1}}{l_{j}}\left(\frac{2 l_{j+1}+1}{2 l_{j}+1}\right)^{v} \leqq\left(\frac{l_{j+1}}{l_{j}}\right)^{v+1} \leqq(1+3 \alpha)^{v+1} .
$$

(b) follows from (a). Proof of (c).

$$
\begin{aligned}
& \frac{\psi_{q+k+2} V_{q+k+2}}{\psi\left(l_{q+k+1}-l_{q+1}+1\right)\left(2 l_{q+k+1}-2 l_{q+1}+3\right)^{v}} \\
& \quad \leqq \frac{l_{q+k+2}}{l_{q+k+1}-l_{q+1}+1} \cdot \frac{\left(2 l_{q+k+2}+1\right)^{v}}{\left(2 l_{q+k+1}-2 l_{q+1}+3\right)^{v}} \\
& \quad \leqq\left(\frac{l_{q+k+2}}{l_{q+k+1}-l_{q+1}}\right)^{v+1}=\left(\frac{l_{q+k+2} / l_{q+k+1}}{1-l_{q+1} / l_{q+k+1}}\right)^{v+1} \leqq\left(\frac{1+3 \alpha}{1-(1+\alpha)^{-k}}\right)^{v+1} \\
& \leqq\left(\frac{1+3 \alpha}{1-(1+\alpha)^{-1}}\right)^{v+1}=\left[\alpha^{-1}(1+\alpha)(1+3 \alpha)\right]^{v+1} .
\end{aligned}
$$

2.4. Lemma. If $P>P_{0}$ is sufficiently large, we have
(a) $\sum_{s:|s|>l_{q}+1-l_{q}} \Psi(|s|) \leqq \frac{A}{4}$,
(b) $\sum_{k=1}^{\infty}\left(\Psi_{k}-\Psi_{k+1}\right) \psi_{q+k+2} V_{q+k+2} \leqq \frac{A}{4}$,
(c) $\frac{1}{8} A \psi_{q} V_{q} \geqq\left(\frac{2 B^{2}}{A}+\frac{A}{8}\right) V_{q+1}$.

We have

$$
\begin{aligned}
(2 l+1)^{v} \psi(l) & =\left[1+\sum_{j=1}^{l}\left((2 j+1)^{v}-(2 j-1)^{v}\right)\right] \psi(l) \\
& \leqq 2 \sum_{j: l / 2 \leqq j \leqq l}\left((2 j+1)^{v}-(2 j-1)^{v}\right) \frac{l}{j} \psi(j) \\
& \leqq 4 \sum_{j: l / 2 \leqq j \leqq l}\left((2 j+1)^{v}-(2 j-1)^{v}\right) \psi(j) \\
& \leqq 4\left[\psi(0)+\sum_{j=1}^{l}\left((2 j+1)^{v}-(2 j-1)^{v}\right) \psi(j)\right] .
\end{aligned}
$$


Therefore

$$
\begin{aligned}
\sum_{l=0}^{\infty} & {[\Psi(l)-\Psi(l+1)] \psi(l)(2 l+1)^{v} } \\
& \leqq 4 \sum_{l=0}^{\infty}[\Psi(l)-\Psi(l+1)]\left[\psi(0)+\sum_{j=1}^{l}\left((2 j+1)^{v}-(2 j-1)^{v}\right) \psi(j)\right] \\
& =4\left[\psi(0) \Psi(0)+\sum_{j=1}^{\infty}\left((2 j+1)^{v}-(2 j-1)^{v}\right) \psi(j) \Psi(j)\right] \\
& =4 \sum_{r \in \mathbb{Z}^{v}} \psi(|r|) \Psi(|r|)<+\infty .
\end{aligned}
$$

Since $q \geqq P$, when $P \rightarrow \infty$ we have $q \rightarrow \infty$ and $l_{q+1}-l_{q} \rightarrow \infty$, proving (a). Using (2.16) we have also

$$
\begin{aligned}
\sum_{k=1}^{\infty}\left(\Psi_{k}-\Psi_{k+1}\right) \psi\left(l_{q+k+1}-l_{q+1}+1\right)\left(2 l_{q+k+1}-2 l_{q+1}+3\right)^{v} & \\
\leqq & \sum_{l=l_{q+2}-l_{q+1}+1}^{\infty}[\Psi(l)-\Psi(l+1)] \psi(l)(2 l+1)^{v} \rightarrow 0 .
\end{aligned}
$$

This, together with part (c) of Lemma 2.3 proves (b). The sequence $\psi_{j}$ increases and tends to infinity, therefore (c) holds as soon as

$$
\psi_{P} \geqq\left(\frac{16 B^{2}}{A^{2}}+1\right)(1+3 \alpha)^{\nu} .
$$

We come now to the proof of Proposition 2.1. We notice first the inequality

$$
\left[\sum_{r} \Psi(|r|)\right]\left(\psi_{q+2} V_{q+2}-\psi_{q} V_{q}\right) \leqq \frac{A}{4} \psi_{q} V_{q}
$$

which follows from Lemma 2.3 (b) and (2.10). Let us write

$$
\begin{aligned}
\sum_{r \in[q+1]} \sum_{s \notin[q+1]} \Psi(|s-r|)\left[n(r)^{2}+n(s)^{2}\right] & \\
= & \sum_{r \in[q+1 \backslash q]} n(r)^{2} \sum_{s \notin[q+1]} \Psi(|s-r|)+\sum_{s \in[q+2 \backslash q+1]} n(s)^{2} \sum_{r \in[q+1]} \Psi(|s-r|) \\
& \quad+\sum_{r \in[q]} n(r)^{2} \sum_{s \notin[q+1]} \Psi(|s-r|)+\sum_{r \in[q+1]} \sum_{s \notin[q+2]} n(s)^{2} \Psi(|s-r|) .
\end{aligned}
$$

Using (2.15), this is

$$
\begin{aligned}
\leqq & {\left[\sum_{r} \Psi(|r|)\right] \sum_{s \in[q+2 \mid q]} n(s)^{2} } \\
& +\left[\sum_{s:|s|>l_{q+1}-l_{q}} \Psi(s)\right] \sum_{r \in[q]} n(r)^{2}+V_{q+1} \sum_{k=1}^{\infty} \Psi_{k} \sum_{s \in[q+k+2 \mid q+k+1]} n(s)^{2} .
\end{aligned}
$$


Applying now first Lemma 2.2 (a), (b), then (2.17) and Lemma 2.4 (a), (b) we obtain

$$
\begin{aligned}
& \sum_{r \in[q+1]} \sum_{s \notin[q+1]} \Psi(|s-r|)\left[n(r)^{2}+n(s)^{2}\right] \\
& \quad \leqq\left[\sum_{r} \Psi(|r|)\right]\left(\psi_{q+2} V_{q+2}-\psi_{q} V_{q}\right) \\
& +\left[\sum_{s:|s|>l_{q+1}-l_{q}} \Psi(|s|)\right] \sum_{r \in[q+1]} n(r)^{2}+V_{q+1} \sum_{k=1}^{\infty}\left(\Psi_{k}-\Psi_{k+1}\right) \quad \psi_{q+k+2} V_{q+k+2} \\
& \leqq \\
& \frac{A}{4} \psi_{q} V_{q}+\frac{A}{4} \sum_{r \in[q+1]}\left[n(r)^{2}+1\right] .
\end{aligned}
$$

Therefore

$$
\begin{aligned}
& -\sum_{r \in[q+1]}\left[\frac{1}{2} A n(r)^{2}-B n(r)\right] \\
& +\sum_{r \in[q+1]} \sum_{s \notin[q+1]} \Psi(|s-r|)\left[\frac{1}{2} n(r)^{2}+\frac{1}{2} n(s)^{2}\right] \\
\leqq & -\sum_{r \in[q+1]}\left[\frac{3}{8} A n(r)^{2}-B n(r)-\frac{A}{8}\right]+\frac{A}{8} \psi_{q} V_{q} .
\end{aligned}
$$

We use now the inequality

$$
n(r) \leqq \frac{2 B}{A}+\frac{A}{8 B} n(r)^{2}
$$

and then (2.8) and Lemma 2.4 (c) obtaining

$$
\begin{aligned}
& -\sum_{r \in[q+1]}\left[\frac{3}{8} A n(r)^{2}-B n(r)-\frac{A}{8}\right]+\frac{A}{8} \psi_{q} V_{q} \\
\leqq & -\sum_{r \in[q+1]}\left[\frac{1}{4} A n(r)^{2}-\frac{2 B^{2}}{A}-\frac{A}{8}\right]+\frac{A}{8} \psi_{q} V_{q} \\
\leqq & -\frac{1}{8} A \psi_{q} V_{q}+\left(\frac{2 B^{2}}{A}+\frac{A}{8}\right) V_{q+1} \leqq 0 .
\end{aligned}
$$

Proposition 2.1 is proved by (2.18) and (2.19)

2.5. Proposition. Let the interaction $U$ be superstable and lower regular.

(a) Given $X=\left(x_{1}, \ldots, x_{n}, \ldots\right)$, suppose that there exists $q$ such that $q \geqq P$ and $q$ is the largest integer for which

$$
\sum_{r \in[q]} n(X, r)^{2} \geqq \psi_{q} V_{q}
$$


Let $X^{\prime}=\left(x_{1}^{\prime}, \ldots, x_{n^{\prime}}^{\prime}\right)$ consist of the points of $X$ contained in $[q+1]$, and $X^{\prime \prime}=\left(x_{1}^{\prime \prime}, \ldots, x_{n^{\prime \prime}}^{\prime \prime}, \ldots\right)$ be the complementary subsequence of $X$. Then, putting $C=\frac{1}{4} A(1+3 \alpha)^{-v-1}$ we have

$$
\begin{aligned}
& -U\left(X^{\prime}\right)-W\left(X^{\prime}, X^{\prime \prime}\right) \\
\leqq & -\frac{1}{4} A \sum_{r \in[q+1]} n(X, r)^{2}-C \psi_{q+1} V_{q+1} .
\end{aligned}
$$

(b) Let $X=\left(x_{1}, \ldots, x_{n}, \ldots\right)$ satisfy

when $j>k$. Then

$$
\sum_{r \in[j]} n(X, r)^{2} \leqq \psi_{j} V_{j}
$$

$$
\sum_{r \notin \mid k]} \Psi(|r|) n(X, r)^{2} \leqq(1+3 \alpha)^{\nu+1} \sum_{l \geqq l_{k}}[\Psi(l)-\Psi(l+1)] \psi(l)(2 l+1)^{\nu} .
$$

We prove (a). From superstability, lower regularity, and Proposition 2.1 we have

$$
\begin{aligned}
& -U\left(X^{\prime}\right)-W\left(X^{\prime}, X^{\prime \prime}\right) \\
\leqq & -\sum_{r \in[q+1]}\left[A n(X, r)^{2}-B n(X, r)\right] \\
& +\sum_{r \in[q+1]} \sum_{s \notin[q+1]} \Psi(|s-r|)\left[\frac{1}{2} n(X, r)^{2}+\frac{1}{2} n(X, s)^{2}\right] \\
\leqq & -\frac{1}{2} A \sum_{r \in[q+1]} n(X, r)^{2} .
\end{aligned}
$$

On the other hand (2.20) and Lemma 2.3 (a) yield

$$
\frac{1}{4} A \sum_{r \in[q+1]} n(X, r)^{2} \geqq \frac{1}{4} A \psi_{q} V_{q} \geqq C \psi_{q+1} V_{q+1} .
$$

(2.21) follows from (2.24) and (2.25).

We prove (b). Using Lemma 2.3 (a) we have

$$
\begin{aligned}
\sum_{r \notin[k]} \Psi(|r|) n(X, r)^{2} & \leqq \sum_{j \geqq k} \Psi\left(l_{j}\right) \sum_{r \in[j+1 \backslash j]} n(X, r)^{2} \\
& =\sum_{j \geqq k}\left[\Psi\left(l_{j}\right)-\Psi\left(l_{j+1}\right)\right] \sum_{r \in[j+1 \backslash k]} n(X, r)^{2} \\
& \leqq \sum_{j \geqq k}\left[\Psi\left(l_{j}\right)-\Psi\left(l_{j+1}\right)\right] \psi_{j+1} V_{j+1} \\
& \leqq(1+3 \alpha)^{v+1} \sum_{j \geqq k}\left[\Psi\left(l_{j}\right)-\Psi\left(l_{j+1}\right)\right] \psi_{j} V_{j} \\
& \leqq(1+3 \alpha)^{v+1} \sum_{l \geqq l_{k}}[\Psi(l)-\Psi(l+1)] \psi(l)(2 l+1)^{v}
\end{aligned}
$$


2.6. Proposition. We define the correlation functions for the bounded Lebesgue measurable region $\Lambda \subset \mathbb{R}^{v}$ by

$\varrho_{\Lambda}\left(x_{1}, \ldots, x_{m}\right)=Z^{-1} \sum_{n \geqq m} \frac{z^{n}}{(n-m) !} \int_{\Lambda^{n-m}} d x_{m+1} \ldots d x_{n} e^{-\beta U\left(x_{1}, \ldots, x_{n}\right)}$

if $x_{1}, \ldots, x_{m} \in \Lambda,=0$ otherwise. If the interaction $U$ is superstable and lower regular, there exists $\xi$ such that

for all $\Lambda, m, x_{1}, \ldots, x_{m}$.

$$
\varrho_{\Lambda}\left(x_{1}, \ldots, x_{m}\right) \leqq \xi^{m}
$$

This will be proved by induction on $m$. We fix $x_{1}, \ldots, x_{m}$, choose the origin of coordinates of $\mathbb{Z}^{v}$ such that $x_{1} \in \mathscr{Q}(0)$ and write

$$
\varrho_{\Lambda}\left(x_{1}, \ldots, x_{m}\right)=\varrho^{\prime}+\varrho^{\prime \prime} .
$$

Here $\varrho^{\prime}$ is the contribution of those $X=\left(x_{1}, \ldots, x_{n}\right)$ such that, for all $j \geqq P$,

$$
\sum_{r \in[j]} n(X, r)^{2} \leqq \psi_{j} V_{j}
$$

and $\varrho^{\prime \prime}$ is the contribution of the other configurations.

For the configurations satisfying (2.28) we have, using lower regularity, Proposition 2.5 (b), and (2.16),

$$
\begin{aligned}
& -2 W\left(\left(x_{1}\right),\left(x_{2}, \ldots, x_{n}\right)\right) \leqq \sum_{r \in \mathbb{Z}^{v}} \Psi(|r|)\left[1+n(X, r)^{2}\right] \\
\leqq & \sum_{r} \Psi(|r|)+\Psi(0) \sum_{r \in[P]} n(X, r)^{2}+\sum_{r \notin[P]} \Psi(|r|) n(X, r)^{2} \\
\leqq & \sum_{r} \Psi(|r|)+\Psi(0) \psi_{P} V_{P}+(1+3 \alpha)^{v+1} \sum_{l \geqq l_{P}}[\Psi(l)-\Psi(l+1)] \psi(l)(2 l+1)^{v} \\
= & 2 D<+\infty .
\end{aligned}
$$

Therefore

$$
\varrho^{\prime} \leqq e^{\beta D} z \varrho\left(x_{2}, \ldots, x_{m}\right) \leqq e^{\beta D} z \xi^{m-1} .
$$

We write $\varrho^{\prime \prime}$ as a sum over $q$, where $q$ is the largest integer such that

$$
\sum_{r \in[q]} n(X, r)^{2} \geqq \psi_{q} V_{q} \text {. }
$$

Let $N(q)$ be the number of points $x_{1}, \ldots, x_{m}$ contained in $[q+1]$. Using Proposition 2.5 (a) and assuming $\xi \geqq z$, we find

$$
\begin{aligned}
\varrho^{\prime \prime} & \leqq \sum_{q \geqq P} \xi^{m-N(q)} z^{N(q)} \exp \left[-\beta C \psi_{q+1} V_{q+1}\right]\left[\sum_{l=0}^{\infty} \frac{1}{l !}\left(V_{q+1} \lambda^{v} z\right)^{l}\right] \\
& \leqq z \xi^{m-1} \sum_{q \geqq P} \exp \left[-\left(\beta C \psi_{q+1}-\lambda^{v} z\right) V_{q+1}\right]=E z \xi^{m-1}
\end{aligned}
$$


where

$$
E=\sum_{q \geqq P} \exp \left[-\left(\beta C \psi_{q+1}-\lambda^{v} z\right) V_{q+1}\right]
$$

converges because $\psi_{q+1} \rightarrow \infty$.

From (2.30) and (2.31) we obtain

$$
\varrho_{\Lambda}\left(x_{1}, \ldots, x_{m}\right) \leqq\left(e^{\beta D}+E\right) z \xi^{m-1} .
$$

Proposition 2.6 holds therefore with $\xi=\left(e^{\beta D}+E\right) z$, which is $\geqq z$.

2.7. Proposition. Let the interaction $U$ be superstable and lower regular. There exist $\gamma>0$ and $\delta$ real such that the following inequalities hold uniformly in $\Lambda$

$$
\varrho_{\Lambda}(X) \leqq \exp \sum_{r}\left[-\gamma n(X, r)^{2}+\delta n(X, r)\right],
$$

(b) let $\Delta$ be a bounded Lebesgue measurable subset of $\mathbb{R}^{v}$ and $x_{1}, \ldots, x_{m} \in \Delta$, we define

$\varrho_{\Lambda \Delta}^{m}\left(x_{1}, \ldots, x_{m}\right)=Z^{-1} \sum_{n \geqq m} \frac{z^{n}}{(n-m) !} \int_{(\Lambda \backslash \Delta)^{n-m}} d x_{m+1} \ldots d x_{n} e^{-\beta U\left(x_{1}, \ldots, x_{n}\right)}$

if $x_{1}, \ldots, x_{m} \in \Lambda,=0$ otherwise; then

$$
\varrho_{A \Delta}^{m}\left(x_{1}, \ldots, x_{m}\right) \leqq \exp \sum_{r}\left[-\gamma n(X, r)^{2}+\delta n(X, r)\right] .
$$

Clearly (2.26) and (2.33) imply $\varrho_{\Lambda \Delta}^{m} \leqq \varrho_{\Lambda}$. Therefore (b) follows from (a). To prove (a) notice first that, by Proposition 2.6,

$$
\varrho_{A}(X) \leqq \xi^{\sum n(X, r)} .
$$

Therefore if $n(X, r) \leqq \psi_{P}^{1 / 2} V_{P}^{1 / 2}$ for all $r \in \mathbb{Z}^{\nu},(2.32)$ is satisfied provided $\gamma, \delta$ are chosen such that

$$
\log \xi+\gamma \psi_{P}^{1 / 2} V_{P}^{1 / 2} \leqq \delta
$$

We fix $\gamma=\frac{1}{4} \beta A$ and complete the proof of (2.32) by induction on the number of $r \in \mathbb{Z}^{v}$ such that $n(X, r) \neq 0$. Suppose that there is $r_{0} \in \mathbb{Z}^{v}$ such that $n\left(X, r_{0}\right)>\psi_{P}^{1 / 2} V_{P}^{1 / 2}$. Changing the origin of coordinates in $\mathbb{Z}^{v}$ we may take $r_{0}=0$; we have then

$$
\sum_{r \in[P]} n(X, r)^{2}>\psi_{P} V_{P}
$$

Therefore, if we denote by $X_{q}^{\prime}$ the subsequence (of $N(q)$ elements) of $X$ contained in $[q+1]$ and by $X_{q}^{\prime \prime}$ the complementary subsequence, 
Proposition 2.5 (a) yields

$$
\begin{gathered}
\varrho_{\Lambda}(X) \leqq \sum_{q \geqq P} z^{N(q)}\left\{\exp _{r \in[q+1]}\left[-\frac{1}{4} \beta A n(X, r)^{2}\right]\right\}\left\{\exp \left[-\beta C \psi_{q+1} V_{q+1}\right]\right\} \\
\times\left[\sum_{l=0}^{\infty} \frac{1}{l !}\left(V_{q+1} \lambda^{v} z\right)^{l}\right] \varrho_{\Lambda}\left(X_{q}^{\prime \prime}\right)
\end{gathered}
$$

Notice that, by $(2.35), z \leqq \xi \leqq e^{\delta}$; since $N(q)>0$ we may write $z^{N(q)}$ $\leqq z e^{-\delta} e^{N(q) \delta}$. Introducing also the induction hypothesis and $\gamma=\frac{1}{4} \beta A$ in (2.36) we find

$\varrho_{\Lambda}(X)$

$\leqq z e^{-\delta}\left\{\exp \sum_{r}\left[-\gamma n(X, r)^{2}+\delta n(X, r)\right]\right\} \sum_{q \geqq P} \exp \left[-\left(\beta C \psi_{q+1}-\lambda^{v} z\right) V_{q+1}\right]$

$=E z e^{-\delta} \exp \sum_{r}\left[-\gamma n(X, r)^{2}+\delta n(X, r)\right]$

but we have $E z \leqq \xi \leqq e^{\delta}$, concluding the proof.

2.8. Corollary. With the notation and assumptions of Proposition 2.7, the grand canonical probability that

is less than

$$
\sum_{r \in \mathscr{R}} n(X, r)^{2} \geqq N^{2} \operatorname{card} \mathscr{R}
$$

$$
\exp \left[-\left(\gamma N^{2}-\lambda^{v} e^{\delta}\right) \operatorname{card} \mathscr{R}\right] .
$$

Taking $\Delta=\cup_{r \in \mathscr{R}} \mathscr{Q}(r)$ in (2.34), we find indeed that the probability to be estimated is less than

$$
\sum_{l} \frac{1}{l !}\left(\operatorname{card} \mathscr{R} \cdot \lambda^{v}\right)^{l} \exp \left[-\gamma N^{2} \operatorname{card} \mathscr{R}\right]\left(e^{\delta}\right)^{l} .
$$

2.9. Corollary. Let the interaction $U$ be superstable and lower regular. There exist $g>0$ and $d \geqq 0$ such that, if $\Delta$ is any bounded Lebesgue measurable subset of $\mathbb{R}^{v}$ with diameter $L \geqq \lambda$, then the grand canonical probability of finding more than $m$ particles in $\Delta$ is less than

$$
\exp \left[-g \frac{m^{2}}{L^{v}}+d m\right]
$$

We first increase $\Delta$ to a set of the form $\Delta(\mathscr{R})$ and notice that

$$
\sum_{r \in \mathscr{R}} n(X, r)^{2} \geqq \frac{1}{\operatorname{card} \mathscr{R}}\left(\sum_{r \in \mathscr{R}} n(X, r)\right)^{2} \geqq \frac{m^{2}}{\operatorname{card} \mathscr{R}} .
$$


Applying Corollary 2.8 we find that the probability to be estimated is less than

$$
\exp \left[-\gamma \frac{m^{2}}{\operatorname{card} \mathscr{R}}+\lambda^{v} e^{\delta} \operatorname{card} \mathscr{R}\right] \leqq \exp \left[-g \frac{m^{2}}{L^{\nu}}+g^{\prime} L^{\nu}\right] .
$$

But a probability being also less than 1 we may replace this estimate by (2.39).

\section{Thermodynamic Limit for the Pressure}

In this section we prove the existence of the thermodynamic limit for the grand canonical pressure in the case of a superstable and lower regular interaction $U$.

3.1. Lemma. If $\mathscr{R}$ is a finite subset of $\mathbb{Z}^{v}$, we write $\Lambda_{\mathscr{R}}=\cup_{r \in \mathscr{R}} \mathscr{Q}(r)$ and let $Z_{\mathscr{R}}$ be the corresponding grand partition function. Let $N$ be chosen such that

$$
\sum_{l=1}^{\infty} \exp \left[-\left(\gamma N^{2}-\lambda^{\nu} e^{\delta}\right)\right] \leqq \frac{1}{4} .
$$

Suppose that $\mathscr{R}, \mathscr{S}$ are finite subsets of $\mathbb{Z}^{v}$, that $\mathscr{R}^{1}, \mathscr{S}^{1} \subset \mathbb{Z}$ are their first projections and that $r^{1}<s^{1}$ whenever $r^{1} \in \mathscr{R}^{1}, s^{1} \in \mathscr{S}^{1}$. The following inequality then holds

$$
\begin{aligned}
\log Z_{\mathscr{R} \cup \mathscr{S}} \leqq \log Z_{\mathscr{R}}+\log Z_{\mathscr{S}}+\log 2 \\
+\frac{1}{2} \beta N^{2}\left[\sum_{r \in \mathscr{R}} \sum_{s: s^{1} \in \mathscr{S} 1} \Psi(|s-r|)+\sum_{s \in \mathscr{S}} \sum_{r: r^{1} \in \mathscr{R}^{1}} \Psi(|s-r|)\right] .
\end{aligned}
$$

Let $\mathscr{R}(l)=\left\{r \in \mathscr{R}: s^{1}-r^{1} \leqq l\right.$ for some $\left.s^{1} \in \mathscr{S}^{1}\right\}, \mathscr{S}(l)=\left\{s \in \mathscr{S}: s^{1}-r^{1} \leqq l\right.$ for some $\left.r^{1} \in \mathscr{R}^{1}\right\}$. The grand canonical probability that

$$
\sum_{r \in \mathscr{R}(l)} n(X, r)^{2} \geqq N^{2} \operatorname{card} \mathscr{R}(l)
$$

is by Corollary 2.8 , less than $\exp \left[-\left(\gamma N^{2}-\lambda^{v} e^{\delta}\right)\right.$ card $\left.\mathscr{R}(l)\right]$ and similarly with $\mathscr{R}(l)$ replaced by $\mathscr{S}(l)$. Therefore, except for a set of probability less than

$$
\begin{gathered}
\sum_{l^{\prime}=1}^{\infty} \exp \left[-\left(\gamma N^{2}-\lambda^{v} e^{\delta}\right) \operatorname{card} \mathscr{R}\left(l^{\prime}\right)\right] \\
+\sum_{l^{\prime \prime}=1}^{\infty} \exp \left[-\left(\gamma N^{2}-\lambda^{v} e^{\delta}\right) \operatorname{card} \mathscr{S}\left(l^{\prime \prime}\right)\right] \leqq \frac{1}{2}
\end{gathered}
$$


we have for all $l^{\prime}, l^{\prime \prime}$

and hence

$$
\begin{gathered}
\sum_{r \in \mathscr{R}\left(l^{\prime}\right)} n(X, r)^{2} \leqq N^{2} \operatorname{card} \mathscr{R}\left(l^{\prime}\right), \\
\sum_{s \in \mathcal{S}\left(l^{\prime \prime}\right)} n(Y, s)^{2} \leqq N^{2} \operatorname{card} \mathscr{S}\left(l^{\prime \prime}\right)
\end{gathered}
$$

$$
\begin{aligned}
-W & (X, Y) \leqq \frac{1}{2} \sum_{r \in \mathscr{R}} \sum_{s \in \mathscr{S}} \Psi(|s-r|)\left[n(X, r)^{2}+n(Y, s)^{2}\right] \\
& \leqq \frac{1}{2} \sum_{r \in \mathscr{R}} n(X, r)^{2} \sum_{s: s^{1} \in \mathscr{S}^{1}} \Psi(|s-r|)+\frac{1}{2} \sum_{s \in \mathscr{S}} n(Y, s)^{2} \sum_{r: r^{1} \in \mathscr{R}^{1}} \Psi(|s-r|) \\
& \leqq \frac{1}{2} N^{2}\left[\sum_{r \in \mathscr{R}} \sum_{s: s^{1} \in \mathscr{S}^{1}} \Psi(|s-r|)+\sum_{s \in \mathscr{S}} \sum_{r: r^{1} \in \mathscr{R}^{1}} \Psi(|s-r|)\right] .
\end{aligned}
$$

This shows that

$$
\frac{Z_{\mathscr{R}} Z_{\mathscr{S}}}{Z_{\mathscr{R} \cup \mathscr{S}}} \exp \frac{1}{2} \beta N^{2}\left[\sum_{r \in \mathscr{R}} \sum_{s: s^{1} \in \mathscr{S}^{1}} \Psi(|s-r|)+\sum_{s \in \mathscr{S}} \sum_{r: r^{1} \in \mathscr{R}^{1}} \Psi(|s-r|)\right] \geqq \frac{1}{2}
$$

proving the lemma.

3.2. Remark. Repeated application of Lemma 3.1 yields $\log Z_{\mathscr{R}_{1} \cup \cdots \cup \mathscr{R}_{k}}$

$$
\begin{aligned}
& \leqq \sum_{j=1}^{k} \log Z_{\mathscr{R}_{j}}+(k-1) \log 2+\frac{1}{2} \beta N^{2} \sum_{j=1}^{k} \sum_{r \in \mathscr{R}_{j}} \sum_{s: s^{1} \notin \mathscr{R}_{j}^{1}} \Psi(|s-r|) \\
& <\sum_{j=1}^{k}\left[\log Z_{\mathscr{R}_{j}}+\log 2+\frac{1}{2} \beta N^{2} \sum_{r \in \mathscr{R}_{j}} \sum_{s \notin \mathscr{R}_{j}} \Psi(|s-r|)\right] .
\end{aligned}
$$

We may of course also apply Lemma 3.1 with the first coordinate direction replaced by another one; repeated application of this yields

$$
\begin{aligned}
& \log Z_{\mathscr{R}_{1} \cup \cdots \cup \mathscr{R}_{k}} \\
& \quad<\sum_{j=1}^{k}\left[\log Z_{\mathscr{R}_{j}}+\log 2+\frac{1}{2} v \beta N^{2} \sum_{r \in \mathscr{R}_{j}} \sum_{s \notin \mathscr{R}_{j}} \Psi(|s-r|)\right] .
\end{aligned}
$$

3.3. Theorem. Given $a^{1}>0, \ldots, a^{v}>0$ and $a=\left(a^{1}, \ldots, a^{v}\right)$ we write

$$
\Lambda^{a}=\left\{x \in \mathbb{R}^{v}: 0 \leqq x^{i}<a^{i} \text { for } i=1, \ldots, v\right\}
$$

and let $Z^{a}$ be the grand partition function computed for the region $\Lambda^{a}$. If the interaction $U$ is superstable and lower regular,

$$
\left|\Lambda^{a}\right|^{-1} \log Z^{a}
$$

converges to a finite limit $\beta p$ when $a^{1}, \ldots, a^{v} \rightarrow \infty$. 
Since $Z$ is an increasing function of $A$, it suffices to prove the theorem for a of the form $l \lambda$ where the components $l^{1}, \ldots, l^{v}$ of $l$ are integers $>0$. We write

$$
\Lambda^{l \lambda}=\Lambda(l), \quad Z^{l \lambda}=Z(l) .
$$

Let

$$
\pi=\liminf _{l \rightarrow \infty}\left(\prod_{1}^{v} l^{i}\right)^{-1} \log Z(l) .
$$

We shall arrive at a contradiction by assuming that the sequence $\left(l_{\alpha}\right)$ tends to infinity and that

$$
\lim _{\alpha \rightarrow \infty}\left(\prod_{1}^{v} l_{\alpha}^{i}\right)^{-1} \log Z\left(l_{\alpha}\right)=\pi^{\prime}>\pi .
$$

Write $\varepsilon=\pi^{\prime}-\pi$. We may, according to (3.4) choose $l_{0}$ such that

$$
\begin{gathered}
\left(\prod_{1}^{v} l_{0}^{i}\right)^{-1} \log Z\left(l_{0}\right)<\pi+\frac{1}{2} \varepsilon, \\
\left(\prod_{1}^{v} l_{0}^{i}\right)^{-1}\left[\log 2+\frac{1}{2} \nu \beta N^{2} \sum_{r \in \Lambda\left(l_{0}\right)} \sum_{s \notin \Lambda\left(l_{0}\right)} \Psi(|s-\mathrm{r}|)\right]<\frac{\varepsilon}{2} .
\end{gathered}
$$

Because $Z$ is an increasing function of $\Lambda$, we may modify $\left(l_{\alpha}\right)$ such that $l_{\alpha}^{i}$ is now a multiple of $l_{0}^{i}$ for all $i, \alpha$ and

$$
\liminf _{\alpha \rightarrow \infty}\left(\prod_{1}^{v} l_{\alpha}^{i}\right)^{-1} \log Z\left(l_{\alpha}\right) \geqq \pi^{\prime}=\pi+\varepsilon
$$

in contradiction with the following inequality derived from (3.3), (3.6) and (3.7)

$$
\begin{aligned}
\left(\prod_{1}^{v} l_{\alpha}^{i}\right)^{-1} & \log Z\left(l_{\alpha}\right) \\
& <\left(\prod_{1}^{v} l_{0}^{i}\right)^{-1}\left[\log Z\left(l_{0}\right)+\log 2+\frac{1}{2} v \beta N^{2} \sum_{r \in \Lambda\left(l_{0}\right)} \sum_{s \notin \Lambda\left(l_{0}\right)} \Psi(|s-r|)\right] \\
& <\pi+\varepsilon .
\end{aligned}
$$

\section{Continuity of the Pressure as a Function of Density}

4.1. Proposition. Let the interaction $U=U_{\Phi}$ be associated with a pair potential $\Phi$ which is superstable and lower regular; assume that

$$
\int d x\left|1-e^{-\beta \Phi(x)}\right|<+\infty .
$$

10 Commun. math. Phys., Vol. 18 
D. Ruelle:

Then there exists $F>0$ independent of $\Lambda$, depending continuously on $\beta$, $z>0$ and such that

$$
\frac{\left\langle n^{2}\right\rangle-\langle n\rangle^{2}}{\langle n\rangle} \geqq \frac{1}{1+F}
$$

where we have introduced the grand canonical average

$$
\left\langle n^{\alpha}\right\rangle=Z^{-1} \sum_{n \geqq 0} n^{\alpha} \frac{z^{n}}{n !} \int d x_{1} \ldots d x_{n} e^{-\beta U\left(x_{1}, \ldots, x_{n}\right)} .
$$

Let us define

$$
\begin{aligned}
\mathscr{M} & =Z^{-1} \sum_{n \geqq 0} \frac{z^{n}}{n !} \int d x_{1} \ldots d x_{n} e^{-\beta U\left(x_{1}, \ldots, x_{n}\right)} \\
& \times \int d y_{1} d y_{2} e^{-\beta W\left(\left(x_{1}, \ldots, x_{n}\right),\left(y_{1}\right)\right)-\beta W\left(\left(x_{1}, \ldots, x_{n}\right),\left(y_{2}\right)\right)}\left(1-e^{-\beta \Phi\left(y_{2}-y_{1}\right)}\right) .
\end{aligned}
$$

4.2. Lemma. Under the conditions of Proposition 4.1 there exists $F>0$ independent of $\Lambda$, depending continuously on $\beta, z>0$ and such that

Let us define

$$
z^{2} \mathscr{M} \leqq F\langle n\rangle
$$

$$
\begin{gathered}
K\left(X,\left(y_{1}, \ldots, y_{n}\right)\right)=\prod_{j=1}^{n} K\left(X, y_{j}\right), \\
K(X, y)=e^{-\beta W(X, y)}-1
\end{gathered}
$$

and note the Mayer-Montroll equations ${ }^{14}$

$\varrho_{\Lambda}(X)=z^{m} e^{-\beta U(X)}\left[1+\sum_{n=1}^{\infty} \frac{1}{n !} \int d y_{1} \ldots d y_{n} K\left(X,\left(y_{1}, \ldots, y_{n}\right)\right) \varrho_{\Lambda}\left(y_{1}, \ldots, y_{n}\right)\right]$.

Using (4.5), (4.6) and Proposition 2.6 we have

$$
\begin{aligned}
\mathscr{M}= & \int_{\Lambda} d y_{1} \int_{\Lambda} d y_{2}\left(1-e^{-\beta \Phi\left(y_{2}-y_{1}\right)}\right) \\
& \times \sum_{n=0}^{\infty} \frac{1}{n !} \int d x_{1} \ldots d x_{n} K\left(\left(y_{1}, y_{2}\right),\left(x_{1}, \ldots, x_{n}\right)\right) \varrho_{\Lambda}\left(x_{1}, \ldots, x_{n}\right) \\
& \leqq|\Lambda|\left[\int d y\left|e^{-\beta \Phi(y)}-1\right|\right] \exp \left[\xi\left(1+e^{2 \beta B}\right) \int d y\left|e^{-\beta \Phi(y)}-1\right|\right]
\end{aligned}
$$

We prove now that $|\Lambda|$ is bounded by a constant multiple of $\langle n\rangle$. Since $\langle n\rangle$ is an increasing function of $z$, it suffices to consider small values of $z$. In particular, since $\lim _{z \rightarrow 0} \xi=0$ according to the estimates of Section 2,

\footnotetext{
${ }^{14}$ See for instance [9].
} 
we may assume that

$$
\begin{gathered}
\left|\sum_{n=1}^{\infty} \frac{1}{n !} \int d y_{1} \ldots d y_{n} K\left((x),\left(y_{1}, \ldots, y_{n}\right)\right) \varrho_{\Lambda}\left(y_{1}, \ldots, y_{n}\right)\right| \\
\leqq \\
\left.\leqq \exp \xi \int d y\left|e^{-\beta \Phi(y)}-1\right|\right\}-1 \leqq \frac{1}{2} .
\end{gathered}
$$

Using (4.9) and (4.7) with $m=1$ we obtain

$$
\langle n\rangle=\int d x \varrho_{\Lambda}(x) \geqq|\Lambda| z\left(1-\frac{1}{2}\right)=\frac{1}{2} z|\Lambda| .
$$

This result, together with (4.8) shows that

$$
z^{2} \mathscr{M} \leqq F\langle n\rangle
$$

for some real $F>0$ (the factor $z^{2}$ is inserted for later convenience).

We come now to the proof of Proposition 4.1, using a method due in its principle to Ginibre [6]. Let the functions $Q$ and $R$ be defined on $\sum_{n \geqq 0} \Lambda^{n}$ by

$$
\begin{gathered}
Q\left(x_{1}, \ldots, x_{n}\right)=n \\
R\left(x_{1}, \ldots, x_{n}\right)=z \int_{\Lambda} d y e^{-\beta W\left(\left(x_{1}, \ldots, x_{n}\right),(y)\right)} .
\end{gathered}
$$

We have, using the Schwarz inequality and Lemma 4.2,

$$
\begin{aligned}
(F+1)^{2}\langle n\rangle^{2} & =\langle F Q+R\rangle^{2} \leqq\left\langle(F Q+R)^{2}\right\rangle \\
& =F^{2}\left\langle n^{2}\right\rangle+2 F\langle n(n-1)\rangle+\left[\langle n(n-1)\rangle+z^{2} \mathscr{M}\right] \\
& \leqq(F+1)^{2}\left\langle n^{2}\right\rangle-(F+1)\langle n\rangle
\end{aligned}
$$

proving (4.1).

4.3. Theorem. Let the interaction $U=U_{\Phi}$ be associated with a pair potential $\Phi$ which is superstable and lower regular; assume that

$$
\int d x\left|1-e^{-\beta \Phi(x)}\right|<+\infty .
$$

The thermodynamic limit $p$ of the grand canonical pressure $p_{A}$ $=\beta^{-1}|\Lambda|^{-1} \log Z$ is a continuous function of the density $\varrho=\beta z \frac{\partial p}{\partial z}$ (inside of its interval of definition).

Writing $\varrho_{\Lambda}=\beta z \frac{\partial p_{\Lambda}}{\partial z}$, we have

$$
\frac{1}{\beta} \frac{d \varrho_{A}}{d p_{A}}=\left(\frac{d}{d z} \log Z\right)^{-1} \frac{d}{d z} z \frac{d}{d z} \log Z=\frac{\left\langle n^{2}\right\rangle-\langle n\rangle^{2}}{\langle n\rangle}
$$

hence $\frac{1}{\beta} \frac{d \varrho}{d p} \geqq \frac{1}{1+F}$ or $\frac{d p}{d \varrho} \leqq \frac{1}{\beta}(1+F)$. 


\section{Equilibrium Equations for a Classical Continuous System}

We say that a family $\left(\sigma_{\Delta}^{m}\right)$ where $\Delta$ runs over the bounded Lebesgue measurable subsets of $\mathbb{R}^{v}$ and $m$ over the positive integers is a system of density distributions if it satisfies the following conditions.

(D0) $\sigma_{\Delta}^{m}$ is a positive Lebesgue integrable function on $\Delta^{m}$ and $\sigma_{\Delta}^{m}\left(x_{i_{1}}, \ldots, x_{i_{m}}\right)=\sigma_{\Delta}^{m}\left(x_{1}, \ldots, x_{m}\right)$ for every permutation $(1, \ldots, m) \rightarrow\left(i_{1}, \ldots, i_{m}\right)$.

(D 1) Normalization

$$
\sum_{m=0}^{\infty} \frac{1}{m !} \int d x_{1} \ldots d x_{m} \sigma_{\Delta}^{m}\left(x_{1}, \ldots, x_{m}\right)=1 .
$$

(D2) Compatibility: if $\Delta \subset \Delta^{\prime}$, then

$$
\sigma_{\Delta}^{m}\left(x_{1}, \ldots, x_{m}\right)=\sum_{n=0}^{\infty} \frac{1}{n !} \int_{\left(\Delta^{\prime} \backslash \Delta\right)^{n}} d x_{m+1} \ldots d x_{m+n} \sigma_{\Delta^{\prime}}^{m+n}\left(x_{1}, \ldots, x_{m+n}\right) .
$$

In particular (2.33) defines a system of density distributions.

We shall associate with each system of density distributions $\left(\sigma_{\Delta}^{m}\right)$ an (abstract) probability measure $\mu$ on a measurable space $(\mathscr{X}, \mathscr{S})$. We let $\mathscr{X}$ be the space of functions $\boldsymbol{X}$ from $\mathbb{R}^{v}$ to the positive integers such that, for any compact $K \subset \mathbb{R}^{v}$,

$$
\sum_{x \in \mathbf{K}} \boldsymbol{X}(x)<+\infty .
$$

$$
X(x)=0 \text { or } 1 \text { ? }
$$

We consider the topology $\mathscr{T}$ on $\mathscr{X}$ defined by the subbasis $\left(\mathcal{O}_{A K}^{m}\right)^{15}$; here $K$ runs over the compact subsets of $\mathbb{R}^{v}, \Lambda$ over the open sets such that $\Lambda \subset K, m$ runs over the positive integers and

$$
\mathcal{O}_{A K}^{m}=\left\{\boldsymbol{X} \in \mathscr{X}: \sum_{x \in \Lambda} \boldsymbol{X}(x)=\sum_{x \in K} \boldsymbol{X}(x)=m\right\} .
$$

We let $\mathscr{S}$ be the $\sigma$-ring of Borel sets with respect to the topology $\mathscr{T}$. It can be shown that $\mathscr{S}$ is the $\sigma$-ring generated by the sets

$$
\mathscr{W}_{\boldsymbol{B}}^{m}=\left\{\boldsymbol{X} \in \mathscr{X}: \sum_{x \in \boldsymbol{B}} \boldsymbol{X}(x)=m\right\}
$$

where $B$ runs over the bounded Borel subsets of $\mathbb{R}^{v 16}$.

Given $\Delta \subset \mathbb{R}^{v}$ we let

$$
\mathscr{X}_{\Delta}=\{X \in \mathscr{X}: x \notin \Delta \Rightarrow \boldsymbol{X}(x)=0\}
$$

${ }^{15}$ I.e., the open sets of $\mathscr{X}$ are the unions of finite intersections of the sets $\mathcal{O}_{A K}^{m}$. This topology and the Borel structure derived from it have been used by Lanford [10].

${ }^{16}$ This result is easy to derive, but we do not want to go here into the necessary settheoretical details; a proof will be published elsewhere. 
and define $\pi_{\Delta}: \mathscr{X} \rightarrow \mathscr{X}_{\Delta}$ by

$$
\left(\pi_{\Delta} X\right)(x)=\left\{\begin{array}{lll}
X(x) & \text { if } & x \in \Delta, \\
0 & \text { if } & x \notin \Delta .
\end{array}\right.
$$

If $\Delta$ is a Borel subset of $\mathbb{R}^{v}$, then $\mathscr{X}_{\Delta} \in \mathscr{S}$ and $\pi_{\Delta}$ is a measurable mapping $(\mathscr{X}, \mathscr{S}) \rightarrow\left(\mathscr{X}_{\Delta}, \mathscr{S}_{\Delta}\right)$ where $\mathscr{S}_{\Delta}=\left\{S \in \mathscr{S}: S \subset \mathscr{X}_{\Delta}\right\}$. [We have $\mathscr{X}_{\Delta}=\bigcap_{l=1}^{\infty} \mathscr{W}_{B(l)}^{0}$ where $B(l)=\{x \notin \Delta:|x| \leqq l\}$, therefore $\mathscr{X}_{\Delta} \in \mathscr{S}$. Furthermore the $\sigma$-ring $\mathscr{S}_{\Delta}$ is generated by the sets $\mathscr{X}_{\Delta} \cap \mathscr{W}_{B}^{m}$ with $B \subset \Delta$ and $\pi_{\Delta}^{-1}\left(\mathscr{X}_{\Delta} \cap \mathscr{W}_{B}^{m}\right)=\mathscr{W}_{B}^{m}$, so that $\pi_{\Delta}$ is measurable.] We have for all $\boldsymbol{X} \in \mathscr{X}$

$$
\boldsymbol{X}=\pi_{\Delta} \boldsymbol{X}+\pi_{\mathbb{R}^{\nu} \backslash \Delta} \boldsymbol{X}
$$

and this relation identifies $\mathscr{X}$ with $\mathscr{X}_{\Delta} \times \mathscr{X}_{\mathbb{R}^{v} \backslash \Delta}$. It is readily seen that ${ }^{17}$

$$
(\mathscr{X}, \mathscr{S})=\left(\mathscr{X}_{\Delta}, \mathscr{S}_{\Delta}\right) \times\left(\mathscr{X}_{\mathbb{R}^{v} \backslash \Delta}, \mathscr{I}_{\mathbb{R}^{\nu} \backslash \Delta}\right) .
$$

Given any $\Delta \subset \mathbb{R}^{v}$, we define a mapping $\omega_{\Delta}: \sum_{m=0}^{\infty} \Delta^{m} \rightarrow \mathscr{X}$ by $\omega_{\Delta}\left(x_{1}, \ldots, x_{m}\right)=\sum_{i=1}^{m} \varepsilon_{x_{i}}$ where $\varepsilon_{x}(y)$ is 1 when $x=y$ and 0 otherwise; $\omega_{\Delta}$ is continuous and, if $\Delta$ is bounded, its image is $\mathscr{X}_{\Delta}$. Let now $\Delta$ be a bounded Borel subset of $\mathbb{R}^{v}$ and $S \in \pi_{\Delta}^{-1} \mathscr{S}_{\Delta}$; we define

$$
\mu(S)=\sigma_{\Delta}\left(\omega_{\Delta}^{-1} \pi_{\Delta} S\right)
$$

where $\sigma_{\Delta}$ is the measure on $\sum_{m=0}^{\infty} \Delta^{m}$ which has the restriction $\frac{1}{m !} \sigma_{\Delta}^{m}\left(x_{1}, \ldots, x_{m}\right) d x_{1} \ldots d x_{m}$ to $\Delta^{m}$. It follows from (D2) that the r.h.s. of (5.9) is independent of $\Delta$, furthermore (5.9) determines the measure $\mu$ on $(\mathscr{X}, \mathscr{S})$ completely because the ring generated by the $\mathscr{W}_{B}^{m}$ consists of sets of the form $S \in \pi_{\Delta}^{-1} \mathscr{S}_{\Delta}$ and the extension of $\mu$ from this ring to $\mathscr{S}$ is unique (see Halmos [8], Section 13, Theorem A). We have thus associated a measure $\mu$ to every system of density distributions $\left(\sigma_{\Delta}^{m}\right)$. Conversely, $\mu$ determines $\left(\sigma_{\Delta}^{m}\right)$ completely.

If $\boldsymbol{X} \in \mathscr{X}, r \in \mathbb{Z}^{\nu}$, we write $n(\boldsymbol{X}, r)=\sum_{x \in \mathscr{Q}(r)} \boldsymbol{X}(x)$. We say that a measure $\mu$ on $(\mathscr{X}, \mathscr{S})$ is tempered if it satisfies the following condition

(T) $\mu$ is carried by the union over $N$ of the sets

$$
S_{N}=\left\{X \in \mathscr{X}:(\forall l) \sum_{r:|r| \leqq l} n(X, r)^{2} \leqq N^{2}(2 l+1)^{\nu}\right\} .
$$

17 The product of two measurable spaces is defined in Halmos [8], Chapter VII. 
This definition is invariant under linear transformations of $\mathbb{R}^{v} ; S_{N}$ is a Borel subset of $\mathscr{X}$.

5.1. Lemma. We write $\Lambda \rightarrow \infty$ if every bounded subset $\Delta$ of $\mathbb{R}^{v}$ is eventually contained in the bounded Borel set $\Lambda$. If $\mathrm{X} \in \mathscr{X}$ we let $[X]_{\Lambda} \in \sum_{m} \Lambda^{m}$ be such that $\omega_{\Lambda}\left([X]_{\Lambda}\right)=\pi_{\Lambda}(X)$.

Let the interaction $U=U_{\Phi}$ be associated with a pair potential $\Phi$ which is superstable, lower regular, and a Borel function ${ }^{18}$.

If $X \in\left(\mathbb{R}^{v}\right)^{m}$, a Borel function $\boldsymbol{W}(X, \cdot)$ is defined on $S_{N}$ by

$$
\boldsymbol{W}(X, Y)=\lim _{\Lambda \rightarrow \infty} W\left(X,[\boldsymbol{Y}]_{\Lambda}\right) .
$$

The proof is immediate. In view of this result we may now introduce the following condition for a probability measure $\mu$ on $(\mathscr{X}, \mathscr{S})$.

(E) Equilibrium Equations. $\mu$ is tempered and, if $\varphi \in L^{1}(\mathscr{X}, \mu)$ and $\Delta$ is a bounded Borel subset of $\mathbb{R}^{v}$,

$$
\begin{aligned}
& \int_{X} \mu(d \boldsymbol{X}) \varphi(\boldsymbol{X}) \\
& =\sum_{m=0}^{\infty} \frac{z^{m}}{m !} \int_{\Delta^{m}} d x_{1} \ldots d x_{m} \int_{\substack{\mathcal{X} \\
\mathbb{R}^{v} \backslash \Delta \\
\varphi\left(\omega_{\Delta}\left(x_{1}, \ldots, x_{m}\right)+\boldsymbol{Y}\right)}} \mu(d \boldsymbol{Y}) e^{-\beta U\left(x_{1}, \ldots, x_{m}\right)-\beta \boldsymbol{W}\left(\left(x_{1}, \ldots, x_{m}\right), Y_{m}\right)}
\end{aligned}
$$

These equations express that if we map $\left(\sum_{m} \Delta^{m}\right) \times \mathscr{X}_{\mathbb{R}^{\nu} \backslash \Delta}$ onto $\mathscr{X}$ by writing

$$
\mathscr{X}=\omega_{\Delta}\left(\sum_{m} \Delta^{m}\right) \times \mathscr{X}_{\mathbb{R}^{v} \backslash \Delta}
$$

then $\mu$ is the image of $e^{-\beta W}\left[\mu_{1} \times \mu_{2}\right]$ where $\mu_{1}$ has the restriction $\frac{z^{m}}{m !} e^{-\beta U\left(x_{1}, \ldots, x_{m}\right)} d x_{1} \ldots d x_{m}$ to $\Delta^{m}$ and $\mu_{2}$ is the restriction of $\mu$ to $\mathscr{X}_{\mathbb{Z}^{v} \backslash \Delta}$.

5.2. Proposition. Let the interaction $U=U_{\Phi}$ be associated with a pair potential $\Phi$ which is superstable, lower regular, and a Borel function; assume that

$$
\int d x\left|1-e^{-\beta \Phi(x)}\right|<+\infty .
$$

Let $\left(\sigma_{a}^{m}\right)$ be a system of density distributions, and $\mu$ be the associated probability measure. Assume that there exist $\bar{\gamma}>0$ and $\bar{\delta}$ real such that

$$
\sigma_{\Delta}^{m}(X) \leqq \exp \sum_{r}\left[-\bar{\gamma} n(X, r)^{2}+\bar{\delta} n(X, r)\right] .
$$

${ }^{18}$ It is necessary here to assume that $\Phi$ is Borel, but Lebesgue measurability is all that will be needed later for states satisfying the equilibrium equations. 
Then $\mu$ is tempered. Furthermore

(a) Let $X \in \Delta^{m}, \Delta$ bounded, then $\exp \left[-\beta W\left(X,[\cdot]_{\Lambda}\right)\right]$ is bounded uniformly in $X$ and $\Lambda$ by a $\mu$-integrable function. In particular, $\exp [-\beta \boldsymbol{W}(X, \cdot)]$ is $\mu$-integrable.

(b) We define correlation functions by

$$
\varrho\left(x_{1}, \ldots, x_{m}\right)=\sum_{n=0}^{\infty} \frac{1}{n !} \int_{\Delta^{n}} d x_{m+1} \ldots d x_{m+n} \sigma_{\Delta}^{m+n}\left(x_{1}, \ldots, x_{m+n}\right)
$$

for $x_{1}, \ldots, x_{m} \in \Delta$, and we write

$$
\begin{gathered}
K\left(\left(x_{1}, \ldots, x_{m}\right),\left(y_{1}, \ldots, y_{n}\right)\right)=\prod_{j=1}^{n} K\left(\left(x_{1}, \ldots, x_{m}\right), y_{j}\right) \\
K\left(\left(x_{1}, \ldots, x_{m}\right), y\right)=\exp \left[-\beta \sum_{i=1}^{m} \Phi\left(y-x_{i}\right)\right]-1 .
\end{gathered}
$$

If the correlation functions satisfy the Mayer equations ${ }^{19}$

$$
\varrho(X Y)=z^{m} e^{-\beta U(X)-\beta W(X, Y)} \sum_{p=0}^{\infty} \frac{1}{p !} \int d v_{1} \ldots d v_{p} K(X, V) \varrho(Y V)
$$

where $X=\left(x_{1}, \ldots, x_{m}\right), Y=\left(y_{1}, \ldots, y_{n}\right), V=\left(v_{1}, \ldots, v_{p}\right)$, then $\mu$ satisfies the equilibrium equations

(c) Conversely, if $\mu$ satisfies the equilibrium equations, then the correlation functions satisfy the Mayer equations.

From (5.14) we obtain (cf. the proof of Corollary 2.8)

$$
\begin{aligned}
& \mu\left(\left\{X \in \mathscr{X}: \sum_{r:|r| \leqq l} n(X, r)^{2} \leqq N^{2}(2 l+1)^{v}\right\}\right) \geqq 1-\exp \left[-\left(\bar{\gamma} N^{2}-\lambda^{v} e^{\bar{\delta}}\right)(2 l+1)^{v}\right], \\
& \mu\left(S_{N}\right) \geqq 1-\sum_{l=0}^{\infty} \exp \left[-\left(\bar{\gamma} N^{2}-\lambda^{v} e^{\bar{\delta}}\right)(2 l+1)^{v}\right] \\
& \geqq 1-\sum_{l=0}^{\infty}\left\{\exp \left[-\left(\bar{\gamma} N^{2}-\lambda^{v} e^{\bar{\delta}}\right)\right]\right\}^{l+1} \\
& \rightarrow 1 \quad \text { when } N \rightarrow \infty .
\end{aligned}
$$

Therefore $\mu$ is tempered.

To prove (a) we notice that

$$
\exp \left[-\beta W\left(X,[\boldsymbol{Y}]_{\Lambda}\right)\right] \leqq \exp \beta \sum_{r \in \mathscr{R}} \sum_{s \in \mathbb{Z}^{\nu}} n(X, r) n(\boldsymbol{Y}, s) \Psi(|s-r|) .
$$

${ }^{19}$ See Mayer [12]. 
Here $\mathscr{R}=\left\{r \in \mathbb{Z}^{v}: \mathscr{Q}(r) \cap \Delta \neq \emptyset\right\}$. Since there are finitely many possible choices of $n(X, r)$, it suffices to prove that

$$
\exp \left[\beta \sum_{r \in \mathscr{R}} n(X, r) \sum_{s \in \mathbb{Z}^{v}} n(\cdot, s) \Psi(|s-r|)\right]
$$

is $\mu$-integrable or, using Hölder's inequality, that

$$
\exp \left[m \beta \sum_{s \in \mathbb{Z}^{v}} n(\cdot, s) \Psi(|s|)\right]
$$

is $\mu$-integrable. Using the notation of Section 2, we decompose $\mathscr{X}$ into one piece such that for all $j \geqq P$

$$
\sum_{r \in[j]} n(X, r)^{2} \leqq \psi_{j} V_{j}
$$

and for each $q \geqq P$ a piece such that $q$ is the largest integer for which

We have

$$
\sum_{r \in[q]} n(\boldsymbol{X}, r)^{2} \geqq \psi_{q} V_{q} .
$$

$$
\sum_{s \in \mathbb{Z}^{v}} n(\boldsymbol{Y}, s) \Psi(|s|) \leqq \Psi(0) \sum_{s \in[q]} n(\boldsymbol{Y}, s)+\sum_{l \geqq l_{q}}[\Psi(l)-\Psi(l+1)] \sum_{s:|s| \leqq l} n(\boldsymbol{Y}, s)
$$

and therefore (see (2.29) and (5.14))

$$
\begin{aligned}
\int \mu(d \boldsymbol{Y}) \exp [ & \left.m \beta \sum_{s \in \mathbb{Z}^{v}} n(\boldsymbol{Y}, s) \Psi(|s|)\right] \\
& \leqq(\exp m \beta D)\left[1+\sum_{q} e^{-\bar{\gamma} \psi_{q} V_{q}} \sum_{l=0}^{\infty} \frac{1}{l !}\left(V_{q} \lambda^{v}\right)^{l}\left(e^{\bar{\delta}+m \beta \Psi^{(0)}}\right)^{l}\right] \\
& =(\exp m \beta D)\left[1+\sum_{q} \exp \left(-\bar{\gamma} \psi_{q} V_{q}+\lambda^{v} e^{\bar{\delta}+m \beta \Psi(0)} V_{q}\right)\right]<+\infty .
\end{aligned}
$$

The $\mu$-integrability of $\exp [-\beta \boldsymbol{W}(X, \cdot)]$ follows then from Lemma 5.1 and Lebesgue's dominated convergence theorem.

We come to the proof of (b). Notice first that if $x_{1}, \ldots, x_{m} \in \mathbb{R}^{v}$, there is a union of less than $m$ cubes $2(r)$ containing $x_{1}, \ldots, x_{m}$; therefore (5.14) yields

$$
\begin{aligned}
\varrho\left(x_{1}, \ldots, x_{m}\right) & \leqq \sum_{n=0}^{\infty} \frac{1}{n !}\left(m \lambda^{v}\right)^{n}\left(e^{\bar{\delta}}\right)^{m+n} \\
& =\left(e^{\bar{\delta}}\right)^{m} \exp m \lambda^{v} e^{\bar{\delta}}=\bar{\xi}^{m}
\end{aligned}
$$

where $\bar{\xi}=\exp \left(\bar{\delta}+\lambda^{v} e^{\bar{\delta}}\right)$. We shall also use the estimate

$$
\begin{aligned}
\int d y\left|K\left(\left(x_{1}, \ldots, x_{m}\right), y\right)\right| & \\
& \leqq \int d y \sum_{l=1}^{m} \exp \left[-\beta \sum_{j=1}^{l-1} \Phi\left(y-x_{j}\right)\right]\left|e^{-\beta \Phi\left(y-x_{l}\right)}-1\right| \\
& \leqq m e^{2 m \beta B} \int d y\left|e^{-\beta \Phi(y)}-1\right| .
\end{aligned}
$$


We introduce the notation

$$
\oint^{ \pm \Delta} d V=\sum_{p=0}^{\infty} \frac{1}{p !} \int_{\Delta^{p}}( \pm 1)^{p} d x_{1} \ldots d x_{p}
$$

so that (5.18) becomes

$$
\varrho(X Y)=z^{m} e^{-\beta U(X)-\beta W(X, Y)} \oint d V K(X, V) \varrho(Y V) .
$$

Let $\Delta$ be a bounded Lebesgue measurable set and take $x_{1}, \ldots, x_{m} \in \Delta$; given $\varepsilon>0$ we can choose a bounded Lebesgue measurable $\Lambda \supset \Delta$ such that

We have then

$$
\int_{\mathbb{R} \backslash \backslash \Lambda} d y|K(X, y)|<\varepsilon .
$$

$$
\begin{array}{r}
\left|\varrho(X Y)-z^{m} e^{-\beta U(X)-\beta W(X, Y)} \oint_{\oint}^{A} d V K(X, V) \varrho(Y V)\right| \\
\leqq z^{m} e^{(m+2 m n) \beta B} \hat{\oint} d V \sum_{q=1}^{\infty} \frac{1}{q !} \int_{(\mathbb{R} v \backslash \Lambda)^{q}} d v_{1}^{\prime} \ldots d v_{q}^{\prime}
\end{array}
$$

$\left|K(X, V) K\left(X,\left(v_{1}^{\prime}, \ldots, v_{q}^{\prime}\right)\right)\right| \varrho\left(Y V\left(v_{1}^{\prime}, \ldots, v_{q}^{\prime}\right)\right)$

$$
\begin{aligned}
& \leqq z^{m} e^{(m+2 m n) \beta B \bar{\xi}} \bar{\xi}\left\{\exp \left[\bar{\xi} m e^{2 m \beta B} \int d y\left|e^{-\beta \Phi(y)}-1\right|\right]\right\}\left(e^{\varepsilon \bar{\xi}}-1\right) \\
& =M \theta^{n}\left(e^{\varepsilon \bar{\xi}}-1\right)
\end{aligned}
$$

where $M, \theta$ depend on $m$. Dropping the superscript $m$ of $\sigma_{A}^{m}$ we have also

$$
\begin{aligned}
& z^{m} e^{-\beta U(X)-\beta W(X, Y)} \hat{\oint} d V K(X, V) \varrho(Y V) \\
& =z^{m} e^{-\beta U(X)-\beta W(X, Y)} \oint d V K(X, V) \oint d V^{\prime} \sigma_{\Lambda}\left(Y V V^{\prime}\right) \\
& =z^{m} e^{-\beta U(X)-\beta W(X, Y)} \oint d V^{\prime \prime} e^{-\beta W\left(X, V^{\prime \prime}\right)} \sigma_{\Lambda}\left(Y V^{\prime \prime}\right) \\
& =\hat{\oint} d V z^{m} e^{-\beta U(X)-\beta W(X, Y V)} \sigma_{\Lambda}(Y V)
\end{aligned}
$$

(5.23) and (5.24) yield

$$
\left|\varrho(X Y)-\oint^{\Lambda} d V z^{m} e^{-\beta U(X)-\beta W(X, Y V)} \sigma_{\Lambda}(Y V)\right| \leqq M \theta^{n}\left(e^{\varepsilon \bar{\xi}}-1\right) .
$$

Choosing now $\Delta^{\prime}$ such that $\Delta \subset \Delta^{\prime} \subset \Lambda$, we find $\left|\oint^{-\Delta^{\prime}} d V \varrho(X Y V)-\int^{\Lambda \mid \Delta^{\prime}} d V \quad z^{m} e^{-\beta U(X)-\beta W(X, Y V)} \sigma_{\Lambda}(Y V)\right| \leqq M \theta^{n} e^{\theta\left|\Delta^{\prime}\right|}\left(e^{\varepsilon \bar{\xi}}-1\right)$ or $\left|\sigma_{\Delta^{\prime}}(X Y)-\oint^{\Lambda \backslash d^{\prime}} d V z^{m} e^{-\beta U(X)-\beta W(X, Y V)} \sigma_{\Lambda}(Y V)\right| \leqq M \theta^{n} e^{\theta\left|\Delta^{\prime}\right|}\left(e^{\varepsilon \bar{\xi}}-1\right) .(5.25)$ 
Let $\varphi$ be a bounded Borel function on $\mathscr{X}$ such that $\varphi(X)=0$ unless $\sum_{x \in \Delta} \boldsymbol{X}(x)=m$ and $\sum_{x \in \Delta^{\prime} \backslash \Delta} \boldsymbol{X}(x)=n$, then (5.25) yields

$$
\begin{gathered}
\left|\int \mu(d X) \varphi(\boldsymbol{X})-\frac{z^{m}}{m !} \int_{\Delta^{m}} d x_{1} \ldots d x_{m} \int_{\substack{\mathbb{R}_{C} \\
\mathbb{R}^{\prime} \backslash}} \dot{\mu}(d \boldsymbol{Y}) e^{-\beta U(X)-\beta W\left(X,[Y]_{\Lambda}\right)} \varphi\left(\omega_{\Delta}(X)+\boldsymbol{Y}\right)\right| \\
\leqq M \theta^{n} e^{\theta\left|\Delta^{\prime}\right|}\left(e^{\varepsilon \xi}-1\right) \frac{|\Delta|^{m}}{m !} \frac{\left|\Delta^{\prime} \backslash \Delta\right|^{n}}{n !}\|\varphi\|_{\infty}
\end{gathered}
$$

If we let $\Lambda \rightarrow \infty$ and $\varepsilon \rightarrow 0$ and use Lebesgue's dominated convergence theorem we obtain the equilibrium equations (5.12).

To prove (c) we choose again $\varphi$ in (5.12) such that $\varphi(X)=0$ unless $\sum_{x \in \Delta} \boldsymbol{X}(x)=m$ and $\sum_{x \in \Delta^{\prime} \backslash \Delta} \boldsymbol{X}(x)=n$, with $\Delta \subset \Delta^{\prime}$. We write then the r.h.s. of (5.12) as the limit when $\Lambda \rightarrow \infty$ of the expression obtained by replacing $W\left(\left(x_{1}, \ldots, x_{m}\right), \boldsymbol{Y}\right)$ by $W\left(\left(x_{1}, \ldots, x_{m}\right),[\boldsymbol{Y}]_{\Lambda}\right)$. If $X \in \Delta^{m}, Y \in\left(\Delta^{\prime} \backslash \Delta\right)^{n}$, we find

$$
\begin{aligned}
& \sigma_{\Delta^{\prime}}^{m+n}(X Y) \\
& =z^{m} e^{-\beta U(X)-\beta W(X, Y)} \lim _{\Lambda \rightarrow \infty} \oint^{\Lambda \backslash \Delta^{\prime}} d V e^{-\beta W(X, V)} \sigma_{\Lambda}(Y V) \\
& =z^{m} e^{-\beta U(X)-\beta W(X, Y)} \lim _{\Lambda \rightarrow \infty} \oint^{\Lambda \backslash \Delta^{\prime}} d V^{\prime} K\left(X, V^{\prime}\right) \oint^{\Lambda \backslash \Delta^{\prime}} d V^{\prime \prime} \sigma_{\Lambda}\left(Y V^{\prime} V^{\prime \prime}\right)
\end{aligned}
$$

In deriving (5.26) from (5.12) we have used the fact that the convergence as $\Lambda \rightarrow \infty$ is uniform in $X$ and $Y$; this is seen in the r.h.s. of (5.26) using

$$
0 \leqq \oint^{\Lambda \backslash d^{\prime}} d V^{\prime \prime} \sigma_{\Lambda}\left(Y V^{\prime} V^{\prime \prime}\right) \leqq \oint d V^{\prime \prime} \sigma_{\Lambda}\left(Y V^{\prime} V^{\prime \prime}\right)=\varrho_{\Lambda}\left(Y V^{\prime}\right) .
$$

In view of (5.14) and (5.19) we may perform the limit $\left|\Delta^{\prime}\right| \rightarrow 0$ in (5.26), obtaining

$$
\begin{aligned}
\varrho(X Y) & =z^{m} e^{-\beta U(X)-\beta W(X, Y)} \lim _{\Lambda \rightarrow \infty} \oint d V K(X, V) \varrho(Y V) \\
& =z^{m} e^{-\beta U(X)-\beta W(X, Y)} \oint d V K(X, V) \varrho(Y V) .
\end{aligned}
$$

5.3. Corollary. Let $\Phi$ satisfy the conditions of Proposition 5.2. If $\mu$ is a probability measure on $(\mathscr{X}, \mathscr{S})$ the following conditions are equivalent

(a) $\mu$ is tempered and satisfies the equilibrium equations,

(b) $\mu$ is associated with a system of density distributions such that the inequalities (5.14) hold (for some $\bar{\gamma}>0, \bar{\delta}$ real) and the correlation functions satisfy the Kirkwood-Salsburg equations:

$$
\varrho((x) Y)=z e^{-\beta W((x), Y)} \sum_{p=0}^{\infty} \frac{1}{p !} \int d v_{1} \ldots d v_{p} K\left((x),\left(v_{1}, \ldots, v_{p}\right)\right) \varrho\left(Y\left(v_{1}, \ldots, v_{p}\right)\right) .
$$


(b) Same as (b) but with $\bar{\gamma}, \bar{\delta}$ in (5.14) replaced by the constants $\gamma$, $\delta$ of Proposition 2.7 .

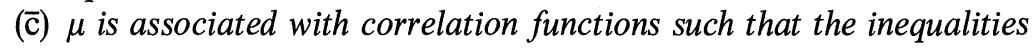

$$
\varrho(X) \leqq \exp \sum_{r}\left[-\bar{\gamma} n(X, r)^{2}+\bar{\delta} n(X, r)\right]
$$

hold ( for some $\bar{\gamma}>0, \bar{\delta}$ real) and the Eqs. (5.27) are satisfied.

(c) same as (c) but with $\bar{\gamma}, \bar{\delta}$ replaced by the constants $\gamma, \delta$ of Proposition 2.7.

If these conditions are satisfied, the correlation functions also satisfy

$$
\varrho\left(x_{1}, \ldots, x_{m}\right) \leqq \xi^{m}
$$

with the constant $\xi$ of Proposition 2.6.

Clearly $(\mathrm{c}) \Rightarrow(\overline{\mathrm{c}}) \Rightarrow(\overline{\mathrm{b}})$ and $(\mathrm{c}) \Rightarrow(\mathrm{b}) \Rightarrow(\overline{\mathrm{b}})$ so there remains to prove $(\bar{b}) \Rightarrow(a),(a) \Rightarrow(c)$ and $(5.28)$.

We notice first that by iteration of the Kirkwood-Salsburg equations (5.27) we obtain the Mayer equations (5.18). Let indeed $X=\left(x_{1}, \ldots, x_{m}\right)$ and use induction on $m$; writing $X^{\prime}=\left(x_{2}, \ldots, x_{m}\right)$ we find

$$
\begin{aligned}
& \varrho(X Y) \\
&=z^{m-1} e^{-\beta U\left(X^{\prime}\right)} e^{-\beta W\left(X^{\prime},\left(x_{1}\right) Y\right)} \oint d V K\left(X^{\prime}, V\right) \varrho\left(\left(x_{1}\right) Y V\right) \\
&=z^{m} e^{-\beta U\left(X^{\prime}\right)} e^{-\beta W\left(X^{\prime},\left(x_{1}\right) Y\right)} e^{-\beta W\left(\left(x_{1}\right), Y\right)} \\
& \times \oint d V e^{-\beta W\left(\left(x_{1}\right), V\right)} K\left(X^{\prime}, V\right) \oint d V^{\prime} K\left(\left(x_{1}\right), V^{\prime}\right) \varrho\left(Y V V^{\prime}\right) \\
&= z^{m} e^{-\beta U(X)} e^{-\beta W(X, Y)} \oint d V^{\prime \prime} K\left(X, V^{\prime \prime}\right) \varrho\left(Y V^{\prime \prime}\right)
\end{aligned}
$$

where we have used

$$
e^{-\beta \Phi\left(v-x_{1}\right)} K\left(X^{\prime}, v\right)+K\left(\left(x_{1}\right), v\right)=K(X, v) .
$$

Therefore $(\bar{b}) \Rightarrow(a)$ by Proposition 5.2 (b).

- Let now $\mu$ satisfy the equilibrium equations. We make the important remark that the estimates of Section 2 for systems enclosed in a bounded region $\Lambda$ also hold for an infinite system described by a tempered probability measure $\mu$ satisfying the equilibrium equations. In fact the estimates in Section 2 were obtained by decomposing the grand canonical probability measure in pieces defined by equations like (2.8), and then applying the equilibrium equations. Since $\mu$ is tempered, the same decompositions and estimates apply to it. In particular, corresponding to Propositions 2.6 and 2.7 we obtain the inequalities (5.28) and

$$
\varrho(X) \leqq \exp \sum_{r}\left[-\gamma n(X, r)^{2}+\delta n(X, r)\right] .
$$

Therefore $(\mathrm{a}) \Rightarrow$ (c) by Proposition 5.2 (c). 
5.4. Remark. We have assumed above that $\Phi$ was a Borel function; it follows from Corollary 5.3 that the meaning of the equilibrium equations is the same for two Borel functions $\Phi$ differing on a set of Lebesgue measure zero. We may thus revert to the point of view that $\Phi$ is a (class of) Lebesgue measurable function and that an arbitrary choice of a Borel function in the class has been made in writing the equilibrium equations.

5.5. Theorem. Let the interaction $U=U_{\Phi}$ be associated with a pair potential $\Phi$ which is superstable and lower regular; assume that

$$
\int d x\left|1-e^{-\beta \Phi(x)}\right|<+\infty .
$$

From every sequence $\left(\Lambda_{l}\right)$ tending to $\infty$ one can extract a subsequence $\left(\Lambda_{l}^{\prime}\right)$ such that (for each $m$ and bounded Lebesgue measurable $\Delta \subset \mathbb{R}^{v}$ ) the following limit exists uniformly in $x_{1}, \ldots, x_{m}$

$$
\lim _{l \rightarrow \infty} \varrho_{\Lambda_{i} \Delta}^{m}\left(x_{1}, \ldots, x_{m}\right)=\sigma_{\Delta}^{m}\left(x_{1}, \ldots, x_{m}\right) .
$$

Furthermore the probability measure $\mu$ associated with the system of density distributions $\left(\sigma_{\Delta}^{m}\right)$ satisfies the equilibrium equations.

If we put on $L^{\infty}\left(\Delta^{m}\right)$ the topology of weak dual of $L^{1}\left(\Delta^{m}\right)$ (with respect to the Lebesgue measure), the set $\left\{\varphi:\|\varphi\|_{\infty} \leqq 1\right\}$ is compact by the theorem of Alaoglu-Bourbaki. In particular, using Proposition 2.7 we find that a subsequence $\left(\Lambda_{l}^{\prime}\right)$ of $\left(\Lambda_{l}\right)$ may be chosen such that (5.29) holds in the sense of convergence in the weak topology of $L^{\infty}\left(\Delta^{m}\right)$ for all $m$ and all $\Delta$ of the form $\left\{x \in \mathbb{R}^{v}:|x|<n\right\}, n$ integer $>0$. But using (D2) and again Proposition 2.7 we see that the convergence holds for arbitrary $\Delta$. Notice that we have also for the correlation functions

$$
\lim _{l \rightarrow \infty} \varrho_{\Lambda_{l}^{\prime}}\left(x_{1}, \ldots, x_{m}\right)=\varrho\left(x_{1}, \ldots, x_{m}\right)
$$

in the weak topology of $L^{\infty}\left(\left(\mathbb{R}^{v}\right)^{m}\right)$. We shall now use the fact that the correlation functions satisfy the Mayer equations ${ }^{20}$

$$
\varrho_{\Lambda}(X Y)=\chi_{\Lambda}(X) z^{m} e^{-\beta U(X)-\beta W(X, Y)} \oint d V K(X, V) \varrho_{\Lambda}(Y V)
$$

where $X=\left(x_{1}, \ldots, x_{m}\right), \chi_{\Lambda}(X)=\prod_{i=1}^{m} \chi_{\Lambda}\left(x_{i}\right)$, and $\chi_{\Lambda}$ is the characteristic function of $\Lambda$. In particular we have the Mayer-Montroll equations

$$
\varrho_{\Lambda}(X)=\chi_{\Lambda}(X) z^{m} e^{-\beta U(X)} \oint d Y K(X, Y) \varrho_{\Lambda}(Y) .
$$

The mapping $\left(x_{1}, \ldots, x_{m}\right) \rightarrow K\left(\left(x_{1}, \ldots, x_{m}\right), \cdot\right)$ is continuous from $\left(\mathbb{R}^{v}\right)^{m}$ to $L^{1}\left(\left(\mathbb{R}^{v}\right)^{n}\right)$ with the norm topology. Therefore the convergence of the functions $\varrho_{A}$ in the weak topology of $L^{\infty}\left(\left(\mathbb{R}^{v}\right)^{n}\right)$ in the r.h.s. of (5.32) as $\Lambda \rightarrow \infty$ implies the convergence of the 1.h.s. uniformly on compacts.

\footnotetext{
${ }^{20}$ See Mayer [12].
} 
We have thus shown that (5.30) holds uniformly on compacts. As a consequence of this (5.29) holds uniformly and (5.14) is satisfied (use Proposition 2.7). Finally, taking $\Lambda \rightarrow \infty$ in (5.31) we obtain (5.18), and the measure $\mu$ associated with $\left(\sigma_{\Delta}^{m}\right)$ satisfies the equilibrium equations by Proposition 5.2 (b).

5.6. Theorem. Under the same assumptions as in Theorem 5.5, the following topologies coincide on the set $\Sigma$ of tempered probability measures satisfying the equilibrium equations,

(a) the topology of uniform convergence of the $\sigma_{\Delta}^{m}$,

(b) the topology of uniform convergence on compacts of the correlation functions,

(c) the topology of convergence of the correlation functions in $L^{\infty}\left(\left(\mathbb{R}^{v}\right)^{m}\right)$ considered as weak dual of $L^{1}\left(\left(\mathbb{R}^{v}\right)^{m}\right)$.

$\Sigma$ is compact for these topologies, and is a simplex in the sense of Choquet.

By Corollary 5.3, every $\mu \in \Sigma$ is associated with a system of density distributions $\left(\sigma_{\Delta}^{m}\right)$ and correlation functions $\varrho$ such that

$$
\varrho(X) \leqq \exp \sum_{r}\left[-\gamma n(X, r)^{2}+\delta n(X, r)\right]
$$

where $\gamma, \delta$ are independent of $\mu$. In view of the relations

$$
\sigma_{\Delta}^{m}\left(x_{1}, \ldots, x_{m}\right)=\sum_{n=0}^{\infty} \frac{1}{n !} \int_{\Delta^{n}}(-1)^{n} d x_{m+1} \ldots d x_{m+n} \varrho\left(x_{1}, \ldots, x_{m+n}\right)
$$

the topologies (a) and (b) are thus equivalent.

Clearly (b) is finer than (c), but since the correlation functions satisfy the equations

$$
\varrho(X)=z^{m} e^{-\beta U(X)} \oint d Y K(X, Y) \varrho(Y)
$$

convergence in the sense of (c) implies convergence in the sense of (b).

Associating with $\mu$ the sequence of its correlation functions, we map $\Sigma$ homeomorphically onto a subset $\Sigma^{*}$ of the (compact) product

$$
\prod_{m=0}^{\infty}\left\{f_{m} \in L^{\infty}\left(\left(\mathbb{R}^{v}\right)^{m}\right):\left\|f_{m}\right\|_{\infty} \leqq \xi^{m}\right\} .
$$

A limit point of $\Sigma^{*}$ again satisfies (5.33) and (5.18), therefore $\Sigma^{*}$ is closed and $\Sigma^{*}, \Sigma$ are compact.

Consider the linear space $\mathscr{L}$ of real measures on $(\mathscr{X}, \mathscr{S})$ which are tempered and satisfy the equilibrium equations. $\Sigma$ is the intersection of the cone $\mathscr{K}$ of positive measures in $\mathscr{L}$ with the hyperplane $\{\mu: \mu(1)=1\}$. Notice that if $\mu \in \mathscr{L}$, then $|\mu| \in \mathscr{L}$ (this follows from the positivity of $e^{-\beta W}$ and the comments after (5.12)). With respect to the usual order on measures 
any two elements $\mu_{1}, \mu_{2}$ of $\mathscr{L}$ have a 1.u.b. $\frac{1}{2}\left(\mu_{1}+\mu_{2}\right)+\frac{1}{2}\left|\mu_{1}-\mu_{2}\right|$ and g.l.b. $\frac{1}{2}\left(\mu_{1}+\mu_{2}\right)-\frac{1}{2}\left|\mu_{1}-\mu_{2}\right|$, these are again in $\mathscr{L}$ and are therefore the l.u.b. and g.l.b. with respect to the order defined in $\mathscr{L}$ by the cone $\mathscr{K}$. Since $\mathscr{L}$ is a lattice for the order defined by $\mathscr{K}, \Sigma$ is a simplex ${ }^{21}$.

5.7. Theorem. Under the same assumptions as in Theorem 5.5, and for sufficiently small $z$ the set $\Sigma$ of tempered probability measures satisfying the equilibrium equations consists of a single point.

If a probability measure satisfies the equilibrium equations, Corollary 5.3 shows that the corresponding correlation functions satisfy (5.27) and (5.28). It is then known that if the following conditions are satisfied

$$
\begin{gathered}
\xi \leqq\left[\int d x\left|e^{-\beta \Phi(x)}-1\right|\right]^{-1}, \\
z<e^{-2 \beta B-1}\left[\int d x\left|e^{-\beta \Phi(x)}-1\right|\right]^{-1}
\end{gathered}
$$

the correlation functions are uniquely determined (see [14], Section 5.2). According to the estimates in Section $2, \xi$ is an increasing continuous function of $z>0$ and $\lim _{z \rightarrow 0} \xi=0$; therefore (5.34) and (5.35) hold for small $z$.

5.8. Theorem. The translations of $\mathbb{R}^{v}$ define a group of homeomorphisms of $\Sigma$. The set $\Sigma_{0}$ of invariant points of $\Sigma$ is non empty, convex and compact; it is a Choquet simplex.

The set $\Sigma_{0}$ is non empty by the theorem of Markov-Kakutani ${ }^{22}$, and is obviously convex and closed. Let $\mathscr{L}_{0}$ be the space of real measures on $(\mathscr{X}, \mathscr{S})$ which are tempered, invariant, and satisfy the equilibrium equations; $\Sigma_{0}$ is the intersection of the cone $\mathscr{K}_{0}$ of positive measures in $\mathscr{L}_{0}$ with the hyperplane $\{\mu: \mu(1)=1\}$. Let $\mu_{+}$and $\mu_{-}$be the 1.u.b. and g.l.b. of $\mu_{1}, \mu_{2} \in \mathscr{L}_{0}$; then $\mu_{+}$and $\mu_{-}$are invariant and therefore belong to $\mathscr{L}_{0}$ (cf. the proof of Theorem 5.6). This shows that $\mathscr{L}_{0}$ is a lattice for the order defined by $\mathscr{K}_{0}$, hence that $\Sigma_{0}$ is a simplex.

\section{References}

1. Bogoljubov, N. N., Khatset, B. I.: On some mathematical questions of the theory of statistical equilibrium. Dokl. Akad. Nauk SSSR 66, 321 (1949).

2. Choquet, G., Meyer, P.-A.: Existence et unicité des représentations intégrales dans les convexes compacts quelconques. Ann. Inst. Fourier 13, 139-154 (1963).

2a. Dobrushin, R. L.: Gibbsian probability field. Funkts. Anal. Ego Pril. 2, 31-43 (1968); 2, 44-57 (1968); 3, 27-35 (1969).

${ }^{21}$ See [2], Theorem 11.

${ }^{22}$ See for instance [4], V. 10.6 (p. 456). 
3. - Minlos, R. A.: Existence and continuity of pressure in classic statistical physics. Teorija Verojatn. i ee Prim. 12, 595-618 (1967).

4. Dunford, N., Schwartz, J. T.: Linear operators. I. General theory. New York: Interscience 1958.

5. Fisher, M. E.: The free energy of a Macroscopic system. Arch. Rat. Mech. Anal. 17, 377-410 (1964).

6. Ginibre, J.: Rigorous lower bound on the compressibility of a classical system. Phys. Letters 24 A, 223-224 (1967).

7. Griffiths, R. B.: Microcanonical ensemble in quantum statistical mechanics. J. Math. Phys. 6, 1447-1461 (1965).

8. Halmos, P. R.: Measure theory. Princeton N. J.: D. Van Nostrand 1950.

9. Hill, T. L.: Statistical mechanics. New York: McGraw-Hill 1956.

10. Lanford, O. E.: The classical mechanics of one-dimensional systems of infinitely many particles. I. An existence theorem. Commun. Math. Phys. 9, 176-191 (1968).

11. - Ruelle, D.: Observables at infinity and states with short range correlations in statistical mechanics. Commun. Math. Phys. 13, 194-215 (1969).

12. Mayer, J. E.: Integral equations between distribution functions of molecules. J. Chem. Phys. 15, 187-201 (1947).

13. Ruelle, D.: Classical statistical mechanics of a system of particles. Helv. Phys. Acta 36, 183-197 (1963).

14. - Statistical mechanics. Rigorous results. New York: Benjamin 1969.

15. van Hove, L.: Quelques propriétés générales de l'intégrale de configuration d'un système de particules avec interaction. Physica 15, 951 - 961 (1949).

16. Yang, C. N., Lee, T. D.: Statistical theory of equations of state and phase transitions. I. Theory of condensation. Phys. Rev. 87, $404-409$ (1952).

D. Ruelle

Institut des Hautes Etudes Scientifiques

F-91 Bures-sur-Yvette

France 